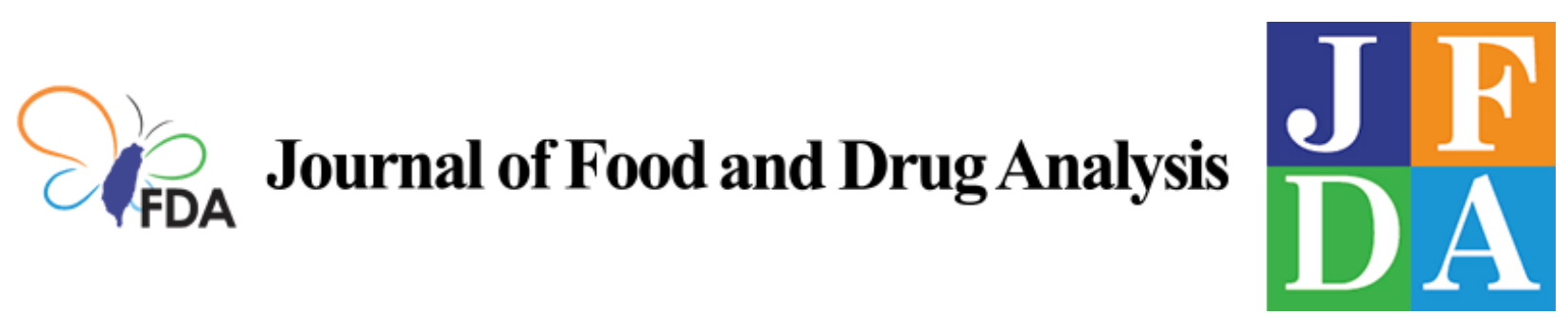

Volume 29 | Issue 4

Article 4

2021

\title{
Medicarpin isolated from Radix Hedysari ameliorates brain injury in a murine model of cerebral ischemia
}

Follow this and additional works at: https://www.jfda-online.com/journal

Part of the Food Science Commons, Medicinal Chemistry and Pharmaceutics Commons, Pharmacology Commons, and the Toxicology Commons

(c) (1) (9)

This work is licensed under a Creative Commons Attribution-Noncommercial-No Derivative Works 4.0 License.

\section{Recommended Citation}

Chern, Chang-Ming; Lu, Chung-Kuang; Liou, Kuo-Tong; Wang, Yea-Hwey; Tsai, Keng-Chang; Chang, ChiaLin; Chang, Chia-Che; and Shen, Yuh-Chiang (2021) "Medicarpin isolated from Radix Hedysari ameliorates brain injury in a murine model of cerebral ischemia," Journal of Food and Drug Analysis: Vol. 29 : Iss. 4 , Article 4.

Available at: https://doi.org/10.38212/2224-6614.3377

This Original Article is brought to you for free and open access by Journal of Food and Drug Analysis. It has been accepted for inclusion in Journal of Food and Drug Analysis by an authorized editor of Journal of Food and Drug Analysis. 


\title{
Medicarpin isolated from Radix Hedysari ameliorates brain injury in a murine model of cerebral ischemia
}

\author{
Chang-Ming Chern c, d,e,1, Chung-Kuang Lu a, Kuo-Tong Liou a,g,h, Yea-Hwey Wang ${ }^{\mathrm{b}}$, \\ Keng-Chang Tsai ${ }^{a}$, Chia-Lin Chang ${ }^{i}$, Chia-Che Chang ${ }^{j, k, 1, m, 1}$, Yuh-Chiang Shen ${ }^{a, b, f, *}$
}

a National Research Institute of Chinese Medicine, Ministry of Health and Welfare, Taipei City, Taiwan
b National Taipei University of Nursing and Health Sciences, Taipei City, Taiwan
${ }^{\text {c }}$ Taipei Municipal Gan-Dau Hospital, Taipei City, Taiwan
d Division of Neurovascular Disease, Neurological Institute, Taipei Veterans General Hospital, Taipei Cit
e Institute of Brain Science, School of Medicine, National Yang Ming Chiao Tung University, Taipei City,
${ }^{f}$ Ph.D. Program in Clinical Drug Development of Herbal Medicine, College of Pharmacy, Taipei Medical Un
g Department of Medicine, Mackay Medical College, New Taipei City, Taiwan
h Department of Chinese Medicine, Tri-Service General Hospital, National Defense Medical Center, Taip
${ }^{\text {i }}$ Research Institute of Biotechnology, HungKuang University, Taichung City, Taiwan
${ }^{j}$ Institute of Biomedical Sciences, Department of Life Sciences, Ph.D. Program in Translational Medicine,
for Translational Medicine, The iEGG and Animal Biotechnology Research Center, National Chung Hsing
Taiwan
k Department of Medical Laboratory Science and Biotechnology, Asia University, Taichung City, Taiwan
${ }^{1}$ Department of Medical Research, China Medical University Hospital, Taichung City, Taiwan
m Traditional Herbal Medicine Research Center, Taipei Medical University Hospital, Taipei City, Taiwan

Abstract

The development of effective post-stroke therapy is highly demanded. Medicarpin is a key active component of a famous Chinese herbal prescription used for post-stroke treatment in Taiwan; however, little is known about its biological effects and mechanisms of action. Herein, we implemented a murine model of cerebral ischemic/reperfusional injury-related stroke to elucidate medicarpin's neuroprotective effect. In male ICR mice $24 \mathrm{~h}$ after stroke induction, treatment with medicarpin $(0.5$ and $1.0 \mathrm{mg} / \mathrm{kg}$, i.v.) markedly enhanced the survival rates, improved moving distance and walking area coverage, reduced brain infarction, and preserved the blood-brain barrier, supporting medicarpin's protective effect on stroke-induced injury. Immunohistochemistry analysis further revealed that medicarpin treatment decreased the expression/activation of p65NF- $B$ and caspase 3, especially near the infarct cortex, while promoting the expression of neurogenesis-associated proteins, including doublecortin (DCX), brain-derived neurotrophic factor (BDNF), and tyrosine receptor kinase B (TrkB). These changes of expression levels were accompanied by GSK-3 inactivation and $\beta$-catenin upregulation. Notably, pretreatment with LY294002, a PI3K inhibitor, abolished the aforementioned beneficial effects of medicarpin, illustrating an essential role of PI3K/Akt activation in medicarpin's neuroprotective and reparative activities. In vitro studies revealed that medicarpin displayed strong anti-inflammatory activity by reducing nitric oxide (NO) production in lipopolysaccharide-stimulated microglial cells (BV2) with an $\mathrm{IC}_{50}$ around $5 \pm 1(\mu \mathrm{M})$ and anti-apoptotic activity in neuronal cells (N2A) subjected to oxygenglucose deprivation with an $\mathrm{IC}_{50}$ around $13 \pm 2(\mu \mathrm{M})$. Collectively, this is the first report to demonstrate that medicarpin, isolated from Radix Hedysari, ameliorates ischemic brain injury through its anti-inflammatory microglia/NO), anti-apoptotic (neuronal cells/OGD) and neuroprotective effects by activating the PI3K/Akt-dependent GSK-3 inactivation for upregulating $\beta$-catenin, which in turn decreases the expression/activation of p65NF- $\kappa$ B and caspase 3 and promotes the expression of neurogenic (DCX, BDNF, TrkB) and neuroprotective $(\mathrm{Bcl} 2)$ factors in the brain.

Keywords: BDNF/TrkB, GSK-3/ $\beta$-catenin, Ischemic stroke, Medicarpin, PI3K/Akt

Abbreviations: $\mathrm{BBB}$, blood-brain barrier; BDNF, brain-derived neurotrophic factor; DCX, doublecortin; GSK-3, glycogen synthase kinase-3; MCAO, middle cerebral artery occlusion; OGD, oxygen and glucose deprivation; PDTC, ammonium pyrrolidinedithiocarbamate; TrkB, tyrosine receptor kinase B.

Received 26 March 2021; revised 14 June 2021; accepted 12 August 2021.

Available online 15 December 2021.

* Corresponding author at: National Research Institute of Chinese Medicine, Ministry of Health and Welfare, 155-1, Linung Street, Sec 2, Peitou, Taipei City, 11221, Taiwan. Fax:+886 228264276.

E-mail address: yuhcs@nricm.edu.tw (Y.-C. Shen).

${ }_{1}$ Chang-Ming Chern and Chia-Che Chang contributed equally to this work. 


\section{Introduction}

A cerebrovascular accident or stroke is a medical emergency that can produce devastating neurological deficits. Stroke is the primary cause of disability worldwide, with an annual mortality rate of around 5.5 million [1]. The pathophysiological mechanisms responsible for ischemic strokeinduced brain injury are highly complex, inducing mitochondrial dysfunction, excitotoxicity, and immune responses. Significantly, during the acute phase of stroke, microglial activation contributes to the post-stroke overproduction of reactive oxygen species (ROS) and inflammatory mediators. In turn, the overproduced ROS and inflammatory mediators cause the loss of neural connections and compromise brain function and blood-brain barrier (BBB) structure, thereby inducing massive oxidative stress and inflammation to provoke necrotic and apoptotic cell death in the brain [2]. To date, thrombolytic recombinant tissue plasminogen activator (rt-PA) is the only FDA-approved clinical treatment for ischemic stroke. However, rt-PA is administered only to a limited group of patients due to its severe side effects (e.g., hemorrhagic transformation) and very narrow therapeutic time window $(<4.5 \mathrm{~h})$ [3]. Therefore, the development of robust and effective post-stroke therapy is still crucial.

To search for neuroprotective strategies, we and many others have underscored that activating the phosphatidylinositol 3-kinase (PI3K)-Akt signaling pathway can reduce ischemic stroke-induced brain injury resulting from the excess release of glutamate (excitotoxicity) [4-7]. It has been revealed that glutamate-induced loss of Akt phosphorylation and activity is most likely caused by the activation of protein phosphatase $2 \mathrm{~A}$ to dephosphorylate Akt through glutamate-induced influx of calcium and sodium ions [8].

Recently, glycogen synthase kinase-3 (GSK-3) has attracted extensive attention as a potential molecular target for drug-mediated neuroprotection against cerebral stroke [9]. GSK-3 is a conserved ubiquitous serine/threonine kinase consisting of $\alpha$ and $\beta$ isoforms. Phosphorylation of Serine (Ser) 21 of GSK- $3 \alpha$ or Ser9 of GSK-3 $\beta$ leads to inhibition of GSK-3 activity [10]. GSK-3 is activated during brain stroke, whereas GSK-3 inactivation, through serine phosphorylation of GSK-3 by PI3K/Akt activation to block glutamate-induced Akt inactivation, protects the brain by promoting angiogenesis, neurogenesis, anti-apoptosis, and anti-inflammation [11]. Also, induction of growth factors such as brain-derived neurotrophic factor (BDNF) is an early and essential step for neuroprotection through stimulating neurogenesis [12]. Tropomyosin-related kinase B receptor $(\operatorname{TrkB})$ is a brain-specific functional receptor of BDNF. Notably, activating the BDNF/TrkB signaling axis has been shown to improve neurobehavior and promote hippocampal neurogenesis in ischemic rats, but its mechanism(s) of action remains unclear [13].

Medicarpin (Fig. 1B) is a natural pterocarpantype phytoalexin [14] with diverse biological activities [15]. It is distributed in numerous traditional Chinese medicines (TCM), including Radix Hedysari [16]. Our previous report demonstrated that a famous TCM prescription for stroke sequelae, $\mathrm{Bu}$ yang Huan-wu decoction (BHD), consists of almost $80 \%$ Radix Hedysari and can effectively protect mice against ischemic stroke by down-regulating factors involved in inflammation and apoptosis along with up-regulating neurogenic factors such as Wnt/frizzled-related protein and doublecortin (DCX) [17]. In the present study, we aimed to examine the neuroprotective and reparative effects of medicarpin, a key active component isolated from Radix Hedysari, in a murine model of acute cerebral ischemic/reperfusional injury (ischemic stroke model). Particularly, we focused on the effect of medicarpin on the GSK-3/ $\beta$-catenin and BDNF/ TrkB signaling pathways in brain protection and neurogenesis promotion after cerebral ischemic injury.

\section{Materials and methods}

\subsection{Preparation of medicarpin}

The dried Hedysarum polybotrys roots (Radix Hedysari) $(4.8 \mathrm{~kg})$, purchased from local herb store (Taipei city, Taiwan), were ground and extracted using ethanol ( 28 L, 3 times) under ambient temperature. After ethanol being evaporated under reduced pressure, the aqueous extract $(2 \mathrm{~L})$ was partitioned with $2 \mathrm{~L}$ ethyl acetate three times. The ethyl acetate layers were combined, dried, and subjected to MPLC column chromatography over C18 gel (Daisogel SP-120 40/60 ODS-B, $240 \times 55 \mathrm{~mm})$ with gradient elution $(5 \%-60 \%$ $\mathrm{MeOH}-\mathrm{H}_{2} \mathrm{O}$ in $10 \mathrm{~min}$ and then $60 \%-100 \%$ $\mathrm{MeOH}-\mathrm{H}_{2} \mathrm{O}$ in $30 \mathrm{~min}, 30 \mathrm{~mL} / \mathrm{min}$ ) to afford fractions $\mathrm{A}-\mathrm{D}$ based on TLC analysis. Fraction $\mathrm{C}$ $(4.66 \mathrm{~g})$ was fractionated by a silica gel column chromatography eluted with $\mathrm{CH}_{2} \mathrm{Cl}_{2} / \mathrm{MeOH}$ stepwise to afford fractions $\mathrm{C} 1-\mathrm{C} 4$. Fraction C2 (1.46 g, $\mathrm{CH}_{2} \mathrm{Cl}_{2} / \mathrm{MeOH}=$ 95:5 eluted) were further purified 
A

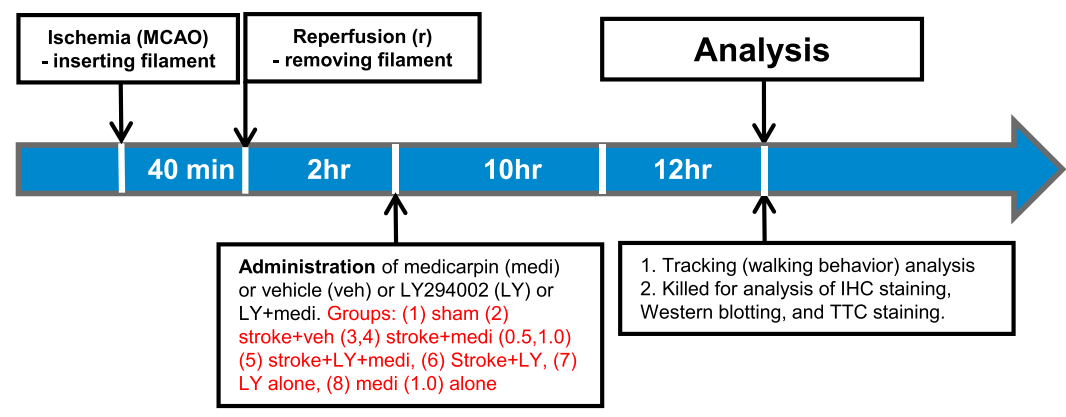

B

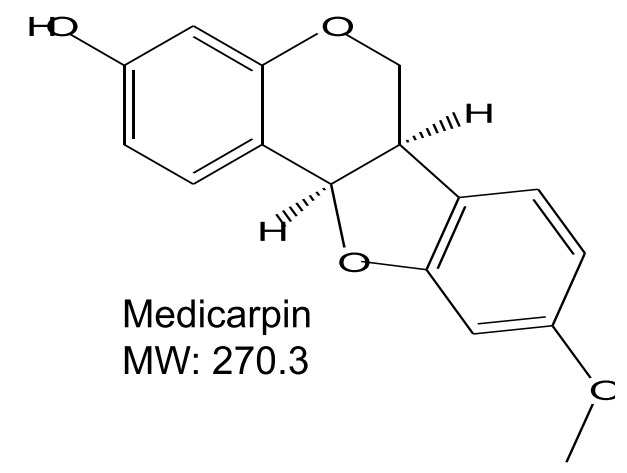

Fig. 1. (A) General experimental protocol and (B) Chemical structure of medicarpin (MW: 270.3).

by C18 HPLC (Cosmosil 5C18 ARII, $5 \mu \mathrm{m}$, $250 \times 10 \mathrm{~mm}, \mathrm{MeCN}-\mathrm{H}_{2} \mathrm{O}, 40: 60,3.0 \mathrm{~mL} / \mathrm{min}$ ) to give medicarpin ( $\left.t_{R} 27.0 \mathrm{~min}, 297.5 \mathrm{mg}\right)$. The structure of medicarpin was determined by NMR and MS data. HRMS (ESI) exact mass calcd for $\mathrm{C}_{16} \mathrm{H}_{15} \mathrm{O}_{4}: 271.0965\left([\mathrm{M}+\mathrm{H}]^{+}\right)$. Found: 271.0962 ([M $\left.+\mathrm{H}]^{+}\right) .{ }^{1} \mathrm{H}$ NMR $\delta_{\mathrm{H}}\left(600 \mathrm{MHz} ; \mathrm{CD}_{3} \mathrm{OD}\right) 3.52-3.55(2$ $\mathrm{H}, \mathrm{m}), 3.74(3 \mathrm{H}, \mathrm{s}), 4.22(1 \mathrm{H}, \mathrm{dd}, \mathrm{J} 2.5,9.3), 5.47(1 \mathrm{H}$, $\mathrm{d}, \mathrm{J}$ 6.5), $6.30(1 \mathrm{H}, \mathrm{d}, \mathrm{J} 2.4), 6.38(1 \mathrm{H}, \mathrm{d}, \mathrm{J} 2.4), 6.45$ (1 $\mathrm{H}, \mathrm{dd}, \mathrm{J} 2.4,8.4), 6.49(1 \mathrm{H}, \mathrm{dd}, \mathrm{J} 2.4,8.4), 7.17(1 \mathrm{H}, \mathrm{d}$, J 8.4), $7.29(1 \mathrm{H}, \mathrm{d}, \mathrm{J} 8.4) ;{ }^{13} \mathrm{C}$ NMR $\delta_{\mathrm{C}}(150 \mathrm{MHz}$; $\left.\mathrm{CD}_{3} \mathrm{OD}\right) 162.8,162.2,160.3,158.2,133.3,126.1,121.0$, 113.0, 110.9, 107.4, 103.2, 97.7, 80.3, 67.7, 56.1, 41.1. The optical rotation angle was $[\alpha]_{\mathrm{D}}^{25}=-218$ (c 1.0, $\mathrm{CH}_{3} \mathrm{OH} ;-188$ (c 0.1, $\mathrm{CHCl}_{3}$ ) [18]). Therefore, its structure was determined to be (-)-3-hydroxy-9ethoxypterocarpan or (-)-medicarpin (Fig. 1B). Its purity was $>98 \%$ by the peak area method at $210 \mathrm{~nm}$ using UHPLC-DAD analysis. Acetonitrile (Merck, German) was of HPLC grade. Ultrapure water was prepared using a Milli- $Q$ water purification system (Millipore, Billerica, MA, USA). All other chemicals and solvents were of analytical grade.
2.2. Animal preparation, induction of an acute cerebral ischemic/reperfusional injury-related ischemic stroke, and drug administration

All animal procedures and protocols were conducted in accordance with The Guide for the Care and Use of Laboratory Animals (NIH publication, 85-23, revised 1996) [19] and were reviewed and approved by the Animal Research Committee of the National Research Institute of Chinese Medicine (approval number: NRICM-IACUC-108-912-1, Feb 21st, 2019). Ischemic stroke injury in male ICR mice (28-30 g, National Laboratory Animal Breeding and Research Center, Taipei, Taiwan) was induced as previously described $[4,20-22]$ in accordance with Stroke Therapy Academic Industry Roundtable recommendations [23]. Briefly, transient focal cerebral ischemia was induced using a heat-blunted nylon monofilament surgical suture (diameter approx. $100 \mu \mathrm{m}$ ) coated with silicone, which was introduced into the exposed external carotid artery, advanced to the internal carotid artery, and wedged into the circle of Willis to obstruct the origin of the right middle cerebral artery (MCA), thereby 
blocking the flow of MCA blood, as determined by transcranial Doppler. The filament was left in place for $40 \mathrm{~min}$ under anesthesia. Following $40 \mathrm{~min}$ of MCA occlusion (MCAO), the filament was withdrawn to achieve reperfusion. The incision was closed and the mice were allowed to recover from anesthesia under a heat lamp to ensure maintenance of a core temperature of $36.0-37.0{ }^{\circ} \mathrm{C}$ during recovery. This procedure leads to reproducible infarcts similar in size and distribution to those reported by others using transient MCAO of comparable duration [24]. Blood samples collected from a femoral artery before MCAO and $30 \mathrm{~min}$ after reperfusion were used for immediate arterial blood gas analysis.

Medicarpin was dissolved in dimethyl sulfoxide (DMSO) to produce $50 \mathrm{mg} / \mathrm{mL}$ stock solution, which was stored at $-20{ }^{\circ} \mathrm{C}$. LY294002 (purity $>98 \%$ by HPLC) was purchased from Sigma-Aldrich. Normal saline with $0.1 \%$ of DMSO was used as a drug reference or vehicle control. To reduce treatment bias, the assignment to the vehicle control and medicarpin treatment groups was randomized and conducted by an investigator unrelated to the study. Throughout the study, the animal treatment groups were blinded to the experimenter and decoded only at the end of the study. Generally, mice were randomly assigned to six groups. These consisted of ( 1 and 2 ) a sham-operated group (sham, $\mathrm{n}=30$ ) and a medicarpin (at $1.0 \mathrm{mg} / \mathrm{kg}$, i.v.)-treated alone without MCAO, (3 and 4) two medicarpin (at $0.5 \mathrm{mg} / \mathrm{kg}$ or $1.0 \mathrm{mg} / \mathrm{kg}$, $2 \mathrm{~h}$ after MCAO, i.v.)treated groups with MCAO (Stroke + M0.5 (or M1.0), $\mathrm{n}=30$, for each dose), and (5 and 6) a vehicle (normal saline with $0.1 \%$ of dimethyl sulfoxide (DMSO))-treated group with MCAO (Stroke + Veh, $\mathrm{n}=30$ ) or a LY294002 (a PI3K inhibitor, at $1.0 \mathrm{mg} / \mathrm{kg}$, i.p., $30 \mathrm{~min}$ before medicarpin treatment) plus medicarpin (at $1.0 \mathrm{mg} / \mathrm{kg}, 2 \mathrm{~h}$ after $\mathrm{MCAO}$, i.v.)treated group with MCAO (Stroke + LY + M1.0, $\mathrm{n}=30$ ). The drug treatment was given once daily over 3 days for the observation of animal survival rates. For other examinations (walking/moving distance/pattern, infarction stains, Western blotting and immunohistochemistry staining), the animals were killed $24 \mathrm{~h}$ after stroke (MCAO) induction; therefore, these animals received the drug treatment only one time after stroke induction. The experimental protocol is presented in Fig. 1A.

\subsection{Evaluation of infarct volume after ischemic stroke}

$24 \mathrm{~h}$ after ischemic stroke, the mice were killed under deep anesthesia. The entire brain was rapidly removed and sliced into $1 \mathrm{~mm}$-thick coronal sections for staining with 2,3,5-triphenyltetrazoliumchloride (TTC). All brain slices were then photographed to determine infarct volume and were corrected for edema, as described in our previous report $[20,21]$.

\subsection{Assessment of neurological deficit}

The neurological deficit of the mice was evaluated immediately prior to sacrifice on day 1 after stroke injury by analyzing their walking distance and a typical stroke-related behavior (walking in circles in a clockwise direction) within $3 \mathrm{~min}$ in a behavior observation box $\left(60 \times 40 \times 60 \mathrm{~cm}^{3}\right)$ using video-tracking system software (SMART v2.5.21, Panlab, Spain) as described in our previous reports $[20,21]$.

\subsection{Immunohistochemical staining}

$24 \mathrm{~h}$ after stroke induction, the brains were prepared for confocal imaging as described in our previous report $[4,20-22]$. Briefly, the whole brains

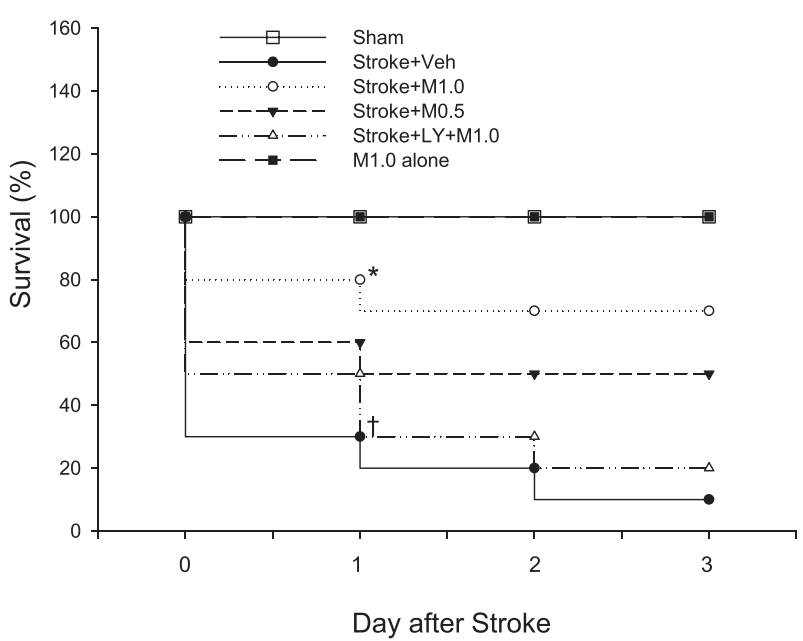

Fig. 2. Effect of medicarpin on the changes in survival rates (over 3 days) after cerebral ischemia-reperfusional injury (stroke). Survival curves were assessed from stroke animals divided into various subgroups as described below using the Log-Rank test followed by the Holm-Sidak method for all pairwise multiple comparisons $(n=10$ for each group in the beginning). ${ }^{*} \dagger p<0.05$, as compared with corresponding vehicle-treated stroke group (Stroke + Veh) or Sham. Animal sub-groups: (1 and 2) a sham-operated group (sham) and a medicarpin (at $1.0 \mathrm{mg} / \mathrm{kg}$, i.v.)-treated alone without stroke induction (M1.0 alone), (3 and 4) two medicarpin (at $0.5 \mathrm{mg} / \mathrm{kg}$ or $1.0 \mathrm{mg} / \mathrm{kg}$, $2 \mathrm{~h}$ after stroke induction, i.v.)-treated groups with stroke (Stroke + M0.5 (or M1.0)), and (5 and 6) a vehicle (normal saline with $0.1 \%$ of dimethyl sulfoxide (DMSO))-treated group with stroke (Stroke + Veh) or a LY294002 (at $1.0 \mathrm{mg} / \mathrm{kg}$, i.p., $30 \mathrm{~min}$ before medicarpin (at $1.0 \mathrm{mg} / \mathrm{kg}$ )) plus medicarpin (at $1.0 \mathrm{mg} / \mathrm{kg}, 2 \mathrm{~h}$ after stroke, i.v.)-treated group with stroke (Stroke + LY + M1.0). 

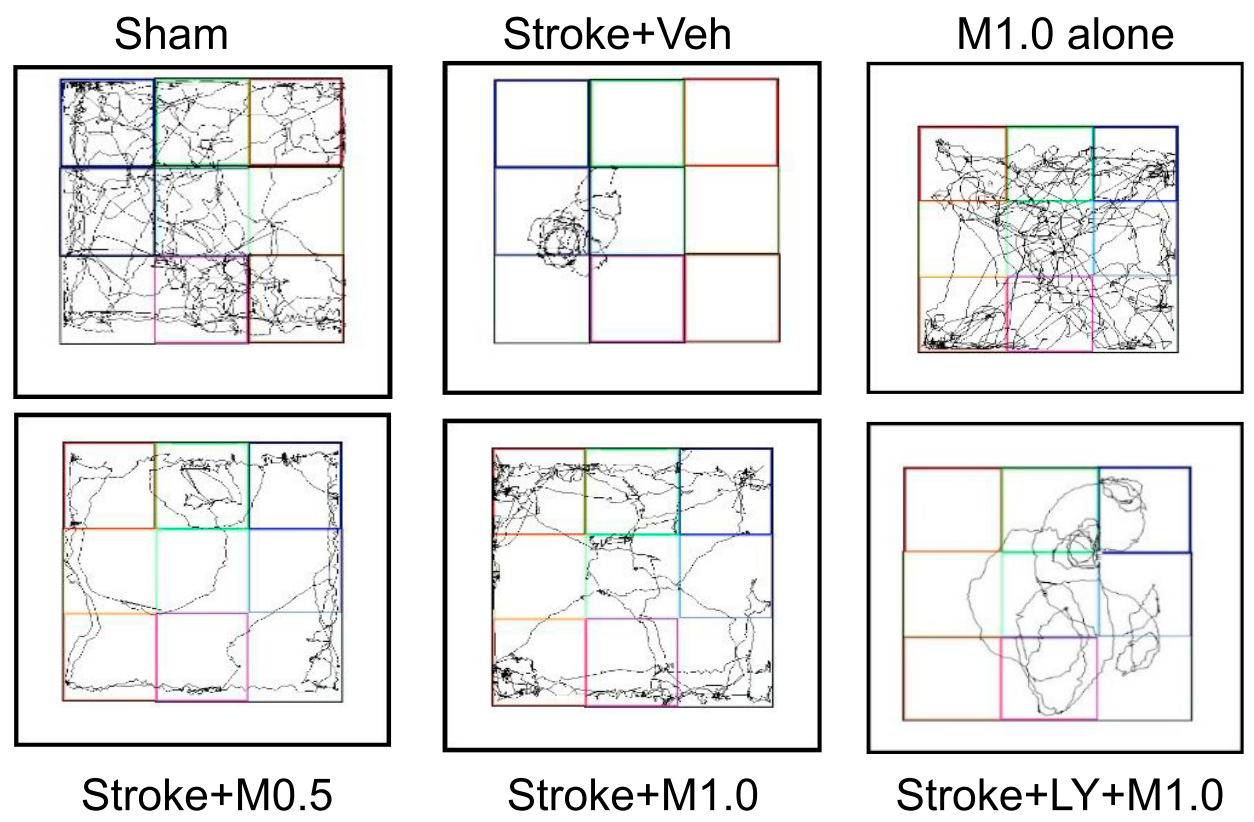

Stroke+M0.5

Stroke+M1.0
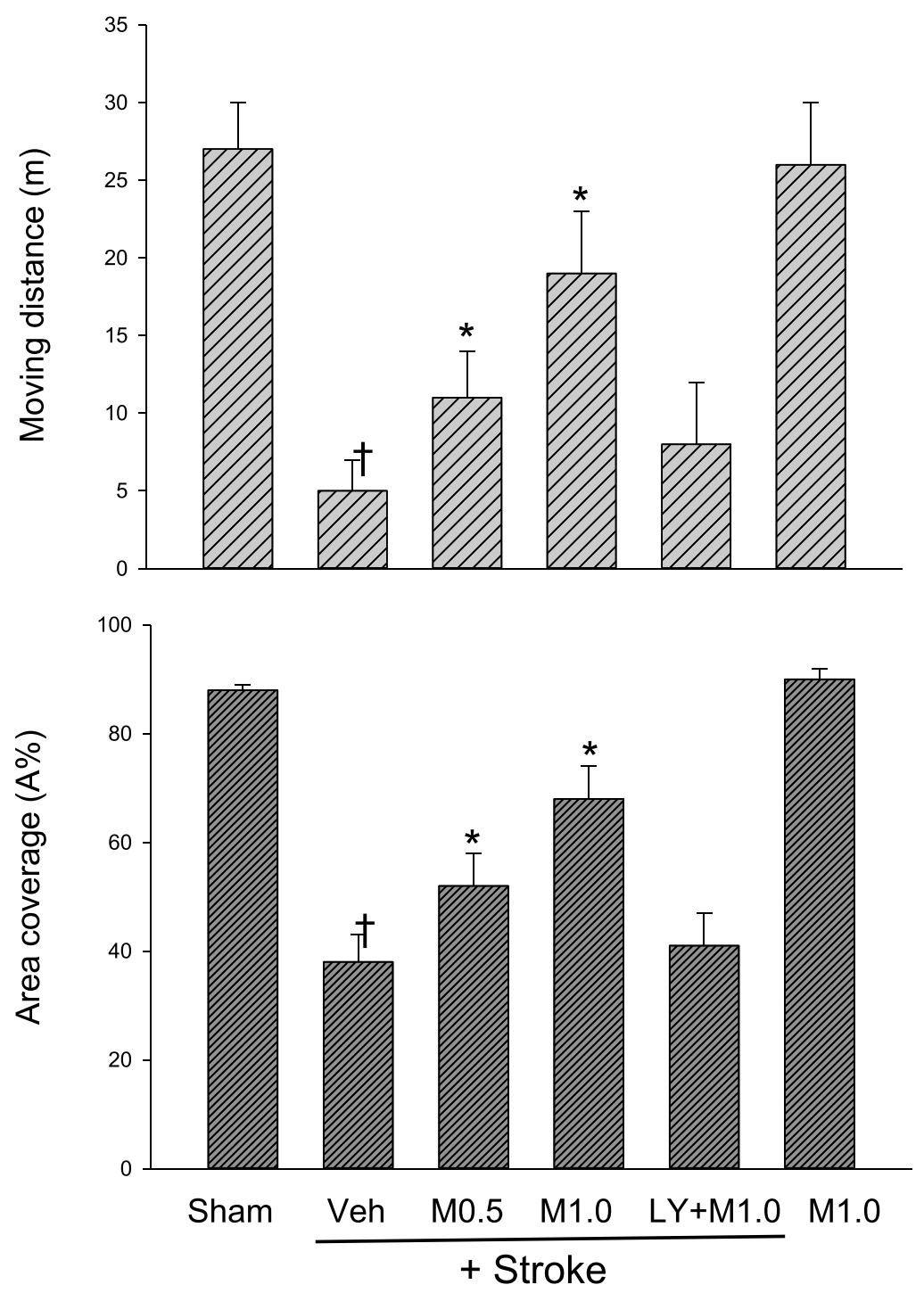
Sham
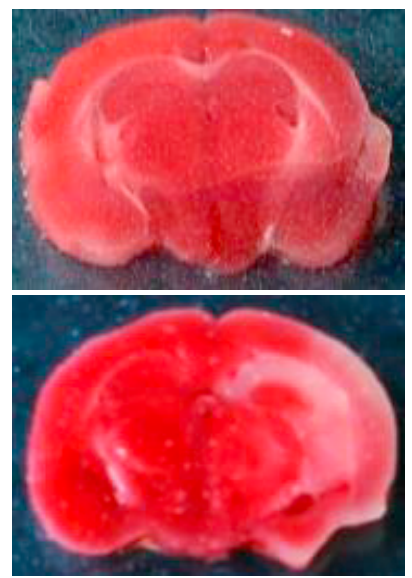

Stroke+M0.5
Stroke+Veh
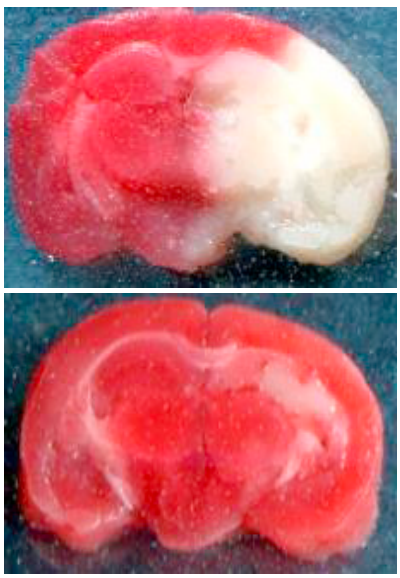

Stroke+M1.0
M1.0 alone
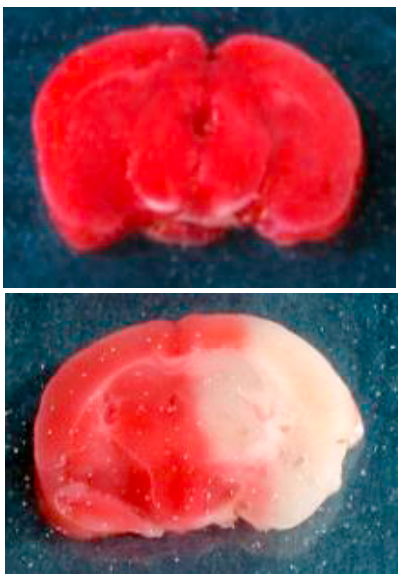

Stroke $+\mathrm{LY}+\mathrm{M} 1.0$

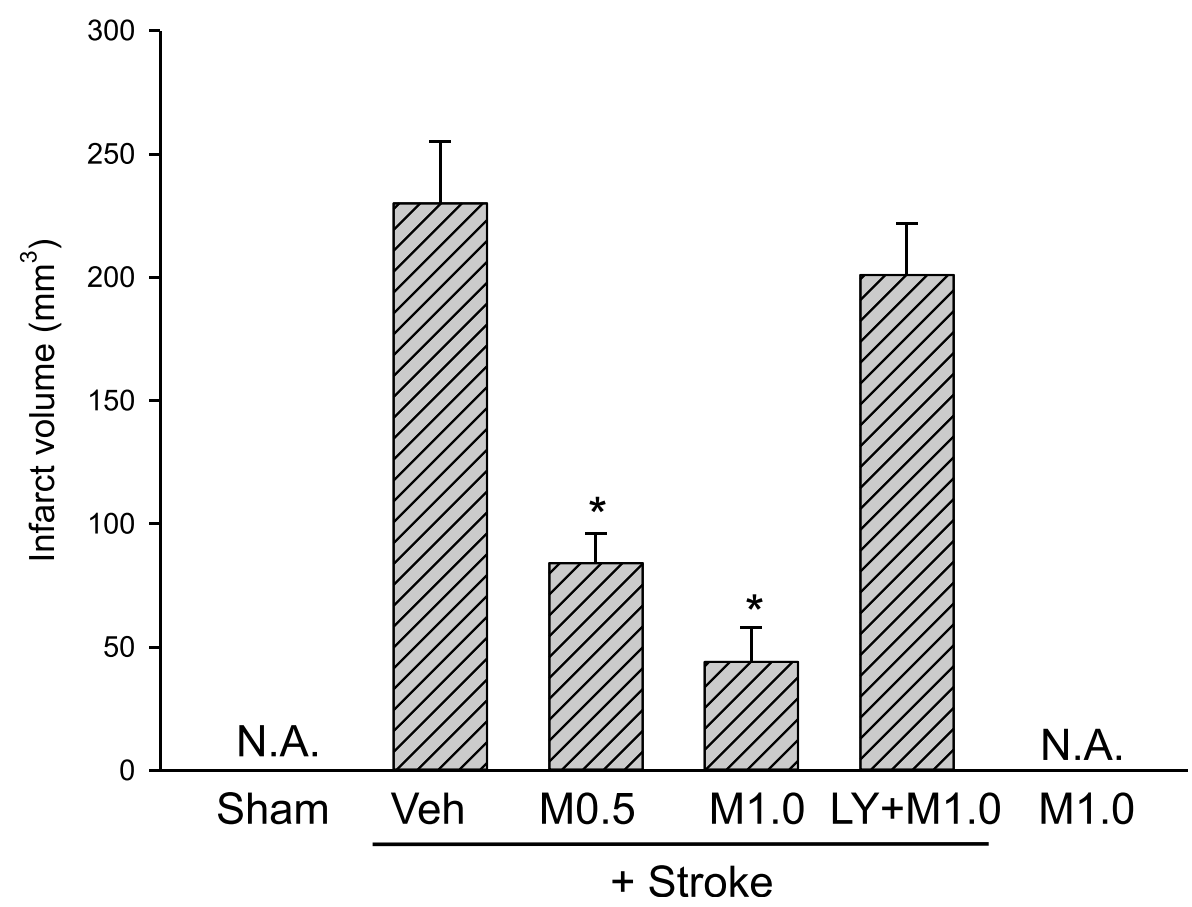

Fig. 4. Effects of medicarpin on the changes in brain infarction $24 \mathrm{~h}$ after cerebral ischemia-reperfusional injury (stroke). Upper panel, typical brain infarction analysis (by TTC stains); lower panel, statistical summary. N.A., data not available. ${ }^{*} p<0.05$, compared with the corresponding vehicletreated stroke (Stroke + Veh) group by one-way ANOVA followed by S-N-K t-test. Animal sub-groups are described in Fig. 2.

were then embedded in Tissue-Tek Optimal Cutting Temperature (OCT) compound (Sakura Finetek,
Torrance, CA USA) for frozen sectioning on a microtome-cryostat. Six consecutive brain sections

Fig. 3. Effects of medicarpin on the changes in neurological deficits (at $24 \mathrm{~h}$ ) after cerebral ischemia-reperfusional injury (stroke). Upper panel, typical animal tracking (moving) profiles within 3 min for evaluation of neurological deficits; statistical results of the neurological deficits analysis were yielded by measuring the moving distance (in meter $(m)$ ) (middle panel) and moving area coverage $(A \%)$ (lower pannel). ${ }^{*} \dagger p<0.05$, compared with the corresponding vehicle-treated stroke (Stroke + Veh) or Sham group by one-way ANOVA followed by S-N-K t-test. Animal sub-groups are described in Fig. 2. 
(thickness, $20 \mu \mathrm{m}$ ) were cut and collected at the same rostrocaudal levels (bregma -1.5 to $-1.9 \mathrm{~mm}$ ) from each group. Following fixation, permeabilization, and blocking, the brain slices were randomly selected for incubation using appropriate primary antibodies against phospho Ser9 GSK-3 $\beta$ (SpGSK-3, 1:50) (Novus Biologicals, CO, USA), occludin (1:100), doublecortin (DCX, 1:100), p65NF- $\kappa \mathrm{B}(1: 50)$, and $\beta$ catenin (catenin, 1:200) (all from Abcam, Cambridge, $\mathrm{UK}$ ), caspase 3 (detecting the active subunit 11 kDa; 1:100; Merck KGaA, Calbiochem, Darmstadt, Germany), and TrkB (1:100, GeneTex, San Antonio, USA) diluted in PBS containing 3\% albumin at $4{ }^{\circ} \mathrm{C}$ overnight. After washing, the sections were incubated with AlexaFluor ${ }^{\circledR 488-}$, AlexaFluor ${ }^{\circledR 555-}$, or AlexaFluor ${ }^{\circledR 647-c o n j u g a t e d ~ s e c o n d-~}$ ary antibodies from Cell Signaling Technology Inc. (MA, USA). All coverslips were mounted using mounting medium containing 4',6-diamidino-2phenylindole (DAPI) to counterstain the DNA in the nuclei. The sliced tissue was examined using a laserscanning confocal microscope (Zeiss LSM780, Carl Zeiss, Jena, Germany). The distribution and number of immuno-positively stained cells were identified and quantified via mean fluorescence intensity (arbitrary units) using imaging software (Zen 2011, black edition, Carl Zeiss MicroImaging $\mathrm{GmbH}$, 1997-2011) across the entire field (10×) of selected images or after sampling in specific regions (e.g., peri-infarct cortex or hippocampus) under high magnification $(60 \times-100 \times)$ over 5 independent experiments.

\subsection{Western blot analysis}

Equal quantities $(20-40 \mu \mathrm{g})$ of protein prepared from the ipsilateral ischemic brain hemispheres (right brain) of the various treatment groups were subjected to $8-12 \%$ sodium dodecyl sulfate-polyacrylamide gel electrophoresis and electro-transferred to a hydrophobic polyvinylidene difluoride membrane, as described in our previous report [4,20-22]. After blocking, the membrane was incubated overnight at $4{ }^{\circ} \mathrm{C}$ with a primary antibody against phospho Ser9 GSK-3 $\beta$ (SpGSK-3, 1:500) (from Santa Cruz Biotechnology, Europe), $\beta$-catenin (1:5000), Bcl-2 (1:1000), occludin (1:2000), BDNF (1:500) (all from Abcam, Cambridge, UK), phospho S473 Akt (pAkt, 1:2000) or $\beta$-actin (1:5000, Sigma-Aldrich, MO, USA). After incubation with a properly titrated secondary antibody, the immunoblot on the membrane became visible following development with an enhanced chemiluminescence
(ECL) system for quantification using imaging software (AlphaEaseFC software, version 4.0, Alpha Innotech Corporation, CA, USA).

\subsection{Induction of neuronal apoptosis by oxygen and glucose deprivation (OGD)}

Our previous protocol was followed [4]. Neuro 2A cells were subjected to oxygen-glucose deprivation (OGD) in the presence of test reagents for $8 \mathrm{~h}$, followed by $16 \mathrm{~h}$ of reperfusion. Quantification of OGD-induced cell apoptosis was measured by Annexin V/propidium iodide assay kits (Invitrogen, CA, USA). AR-a014418 (a GSK-3 inhibitor; purity $>98 \%$, Sigma-Aldrich) also included as a positive control.

\subsection{Assay of LPS-mediated nitric oxide (NO) production in microglial cell (BV-2) and 1,1- diphenyl-2-picrylhydrazyl (DPPH) radical- scavenging capacity}

Our previous methods were followed [4]. Briefly, BV-2 cells were pretreated with test samples for $1 \mathrm{~h}$, followed by addition of lipopolysaccharide (LPS, $500 \mathrm{ng} / \mathrm{mL}$ ) for another $24 \mathrm{~h}$. Nitrite $\left(\mathrm{NO}_{2}\right)$ accumulation in the medium as an indicator of $\mathrm{NO}$ production was measured by Griess reagent. An NF- $\kappa$ B inhibitor, ammonium pyrrolidinedithiocarbamate (PDTC, purity $>99 \%$, Sigma-Aldrich), was included as a reference for the inhibition of NO production. For DPPH radical-scavenging capacity assay, DPPH solution was added to each diluted sample, and the resulting solution was allowed to react for $30 \mathrm{~min}$ in the dark. The absorbance caused by the DPPH radical at $517 \mathrm{~nm}$ was determined with a microplate-spectrophotometer. The radical-scavenging capacity was expressed as delta OD517 ( $\triangle$ OD517). An antioxidant, trolox (purity $>98.4 \%$, OXIS), was included as a reference.

\subsection{Statistical analysis}

All results in the text and figures are presented as mean \pm SEM (standard error of the mean). Data were analyzed using one-way analysis of variance (ANOVA) followed by the post hoc Student-Newman-Keuls (S-N-K) $t$-test for multiple comparisons, using statistical analysis software (SigmaStat, v3.5). Survival rates was analyzed using Log-Rank test followed by the Holm-Sidak method. Values of $p<0.05$ were considered significant. 


\section{Results and discussion}

3.1. Medicarpin improved survival rates and alleviated neurological deficits in mice with cerebral ischemic/reperfusional injury (ischemic stroke)

To determine the protective effect of medicarpin on the ischemic stroke-mediated brain injury, we examined animal survival rates, neurological deficits (moving performance), and brain damage (blood-brain barrier (BBB) damage, infarction and apoptosis) after inducing ischemic stroke in mice. Over $70 \%$ of mice treated with vehicle (normal saline containing $0.1 \%$ DMSO) died within 3 days after ischemic stroke (Fig. 2, Stroke + Veh vs. Sham, $p<$ 0.05). In contrast, treatment of medicarpin $(0.5-1.0 \mathrm{mg} / \mathrm{kg}$, once daily, i.v.) dose-dependently improved the survival rate of the ischemic mice as compared to the vehicle-treated stroke group. This was particularly noteworthy at $24 \mathrm{~h}$ (day 1 ) after ischemic stroke in the mice treated with medicarpin $(1.0 \mathrm{mg} / \mathrm{kg})$ (Fig. 2, $p<0.05$, as compared with Stroke + Veh).

Ischemic stroke induced dramatic neurological deficits in mice treated with vehicle, as evidenced by a reduction in the moving distance (in meter $(\mathrm{m})$ ) (Fig. 3, middle panel, Stroke + Veh vs. Sham, $p<0.05)$ and showing a typical neurological deficit behavior (Fig. 3; upper panel, Stroke + Veh, moving in circles in a clockwise direction) with a significant reduction in the coverage of walking area $(\mathrm{A} \%)$ (Fig. 3, lower panel, Stroke + Veh vs. Sham, $p<0.05)$. On the contrary, medicarpin improved moving distance and neurological deficit behavior (walking in circles and decreased walking area coverage (A\%)) after ischemic stroke (Fig. 3, middle panel, Stroke + Medicarpin (M0.5 or M1.0) vs. Stroke + Veh, $p<0.05)$.

\subsection{Medicarpin ameliorated cerebral infarction, $B B B$ damage, inflammation, and apoptosis in mice after ischemic stroke}

We reported previously that after cerebral ischemic stroke, the brain tissue dramatically lost occludin staining along with a strong leakage of rhodamine B isothiocyanate (RhITC, a fluorescent dye) staining, indicating severe damage of the BBB structure $[20,21]$. Also, it has been demonstrated that increased p65NF- $\kappa \mathrm{B}$ levels in the ischemic damaged (infarct) area correlate well with the activation/nuclear translocation of p65NF- $\kappa \mathrm{B}$ and the up-regulation of p65NF- $\kappa \mathrm{B}$ 's downstream inflammatory proteins such as iNOS and COX-2 [20,21].
Therefore, we evaluated BBB and tissue damage by the loss of occludin staining and assessed brain inflammation by the increased levels of p65NF- $\kappa$ B. It is noteworthy that ischemic stroke led to robust cerebral infarction (loss of 2,3,5-triphenyltetrazoliumchloride (TTC) staining and thus turning white color) (Fig. 4, Stroke + Veh), BBB and tissue damage (Fig. 5A, loss of occludin (green) staining, Sham vs. Stroke + Veh, $p<0.05$ ), inflammation (Fig. 5A, increased levels of p65NF- $\mathrm{B}$ (red), Sham vs. Stroke + Veh, $p<0.05$ ), and apoptosis (Fig. 5B and $E$, increased levels of the active form of caspase3 (red) staining, Sham vs. Stroke + Veh, $p<0.05$ ). Significantly, treatment of ischemic stroke mice with medicarpin $(0.5-1.0 \mathrm{mg} / \mathrm{kg}$, i.v.) reduced the levels of cerebral infarction (Fig. 4, Stroke + Medicarpin (M0.5 or M1.0) vs. Stroke + Veh, $p<0.05$ ), BBB and tissue damage, inflammation, and apoptosis (Fig. 5A and B, Stroke + Medicarpin (M0.5 or M1.0) vs. Stroke + Veh, $p<0.05)$.

\subsection{Medicarpin promoted endogenous neurogenesis in mice after ischemic stroke}

To explore whether endogenous neurogenesis, typically around the dentate gyrus (hippocampus), could be promoted by medicarpin at $24 \mathrm{~h}$ after ischemic injury, we used doublecortin (DCX, a marker for newborn neuroblasts/neural progenitor cells) $[20,21]$ as an indicator of endogenous neurogenesis. Compared to the sham-operated group, the levels of DCX (orange)-positively stained cells were intensely reduced in mice treated with the vehicle after ischemic injury (Fig. 5C, Stroke + Veh vs. Sham, $p<0.05)$. In contrast, as compared to the vehicle-treated group, medicarpin dramatically upregulated DCX-positively stained cells in a dosedependent manner (Fig. 5C, Stroke + Veh vs. Stroke + M0.5 (M1.0), p < 0.05). More importantly, most of these DCX-positive cells displayed clear neurite outgrowth and integrated well into the granule cell layer of dentate gyrus (indicated by arrows in Fig. 5C, Stroke + M1.0). Overall, these results highlight the promoting effect of medicarpin on the formation of a new network of synaptic connections by those DCX-positive neurons within the damaged area.

\subsection{Medicarpin promoted endogenous neurogenesis in mice after ischemic stroke through GSK-3 inhibition and $\beta$-catenin activation}

To elucidate the possible neuronal protective signal(s) initiated by medicarpin, we examined whether medicarpin modulates the GSK-3/ $\beta$ - 
A
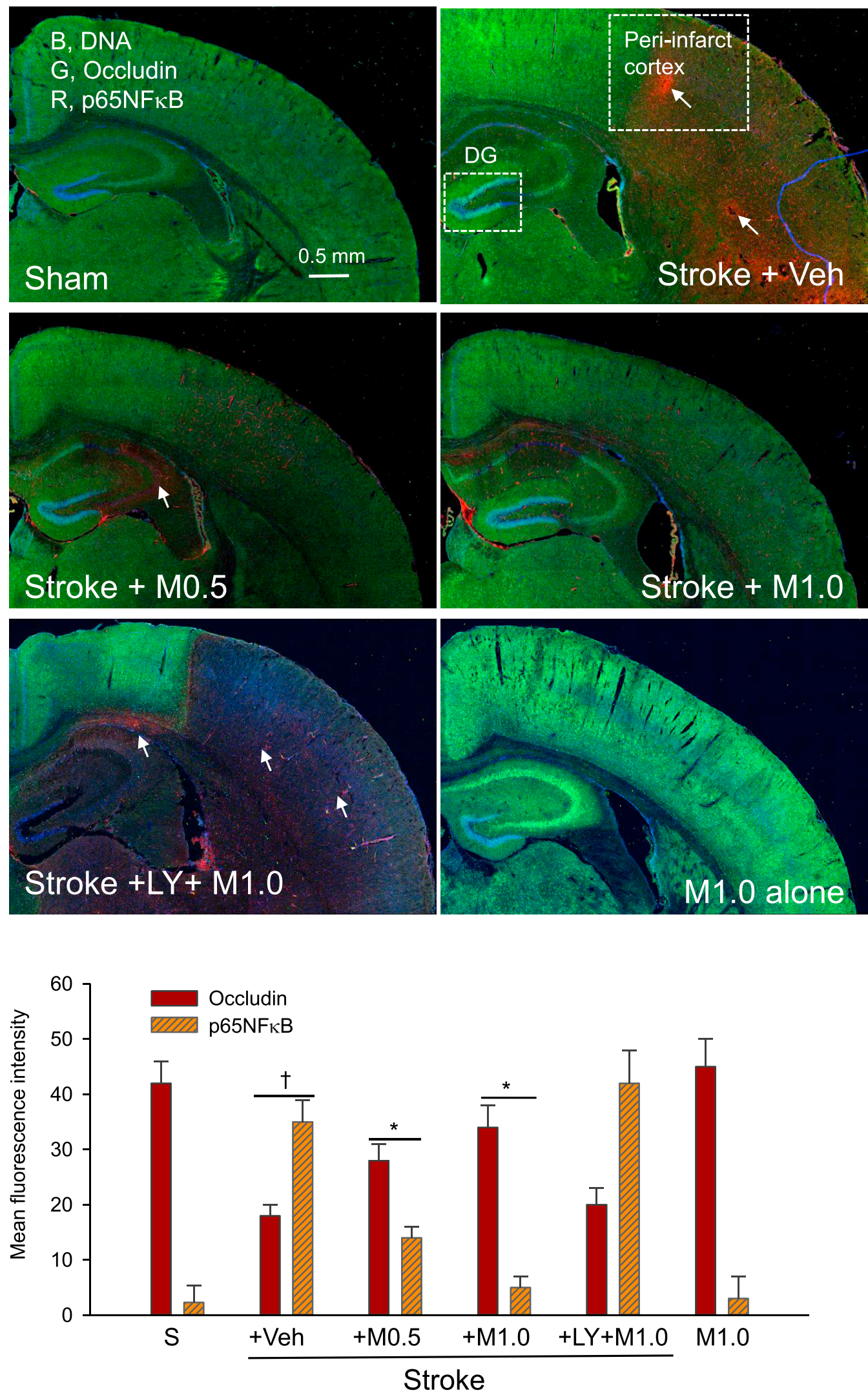


\section{B}
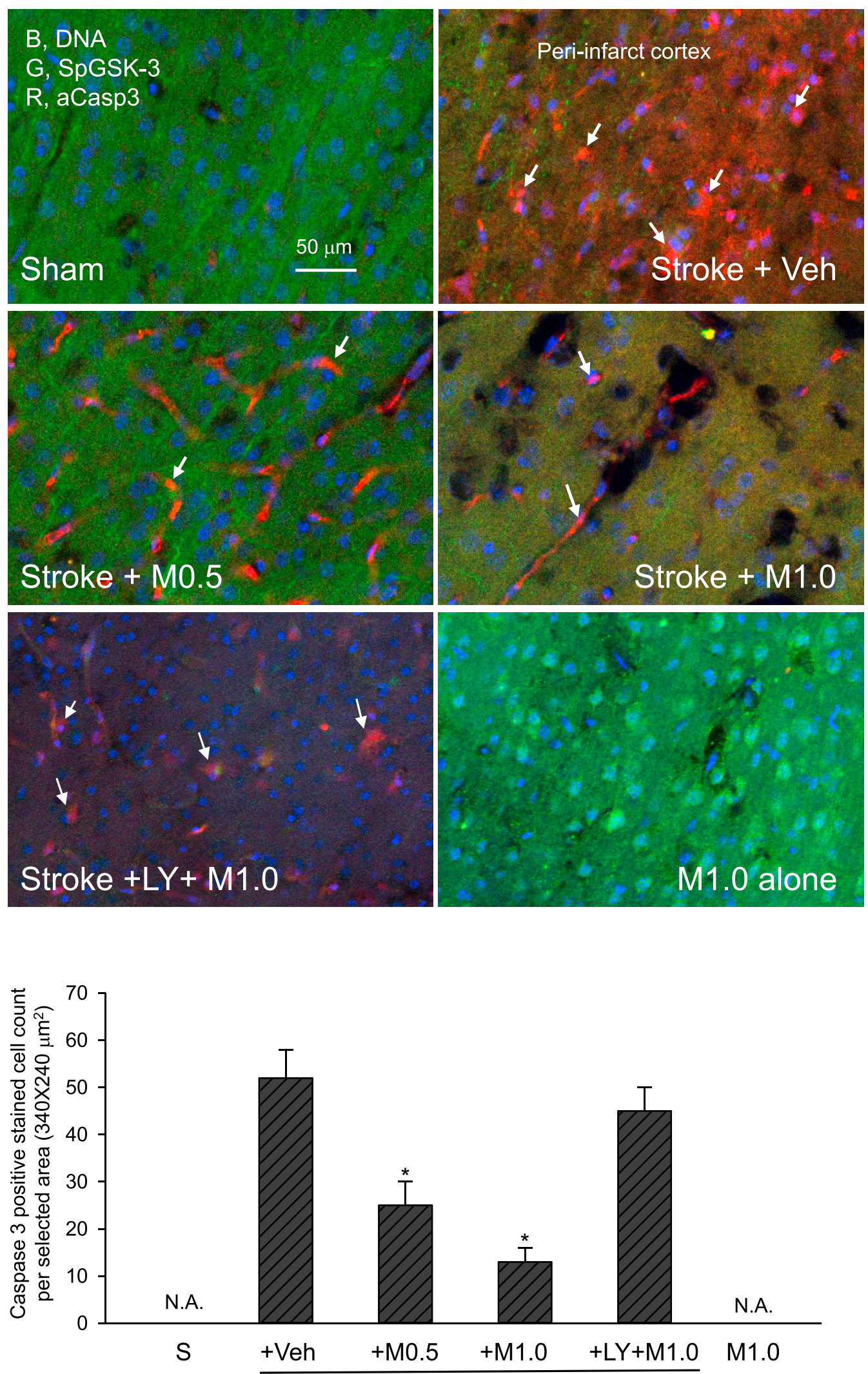
C
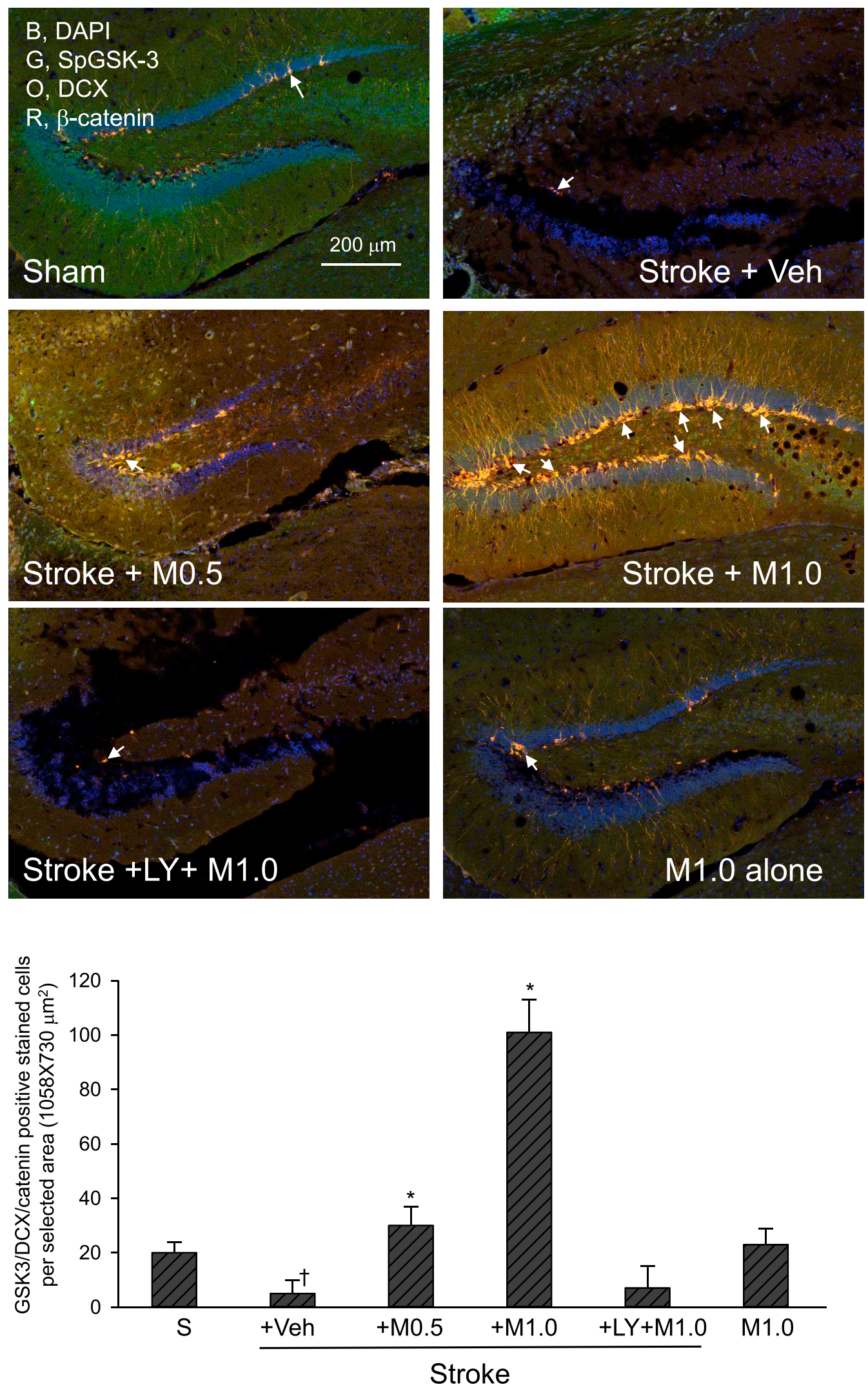

Fig. 5. (Continued). 


\section{D}
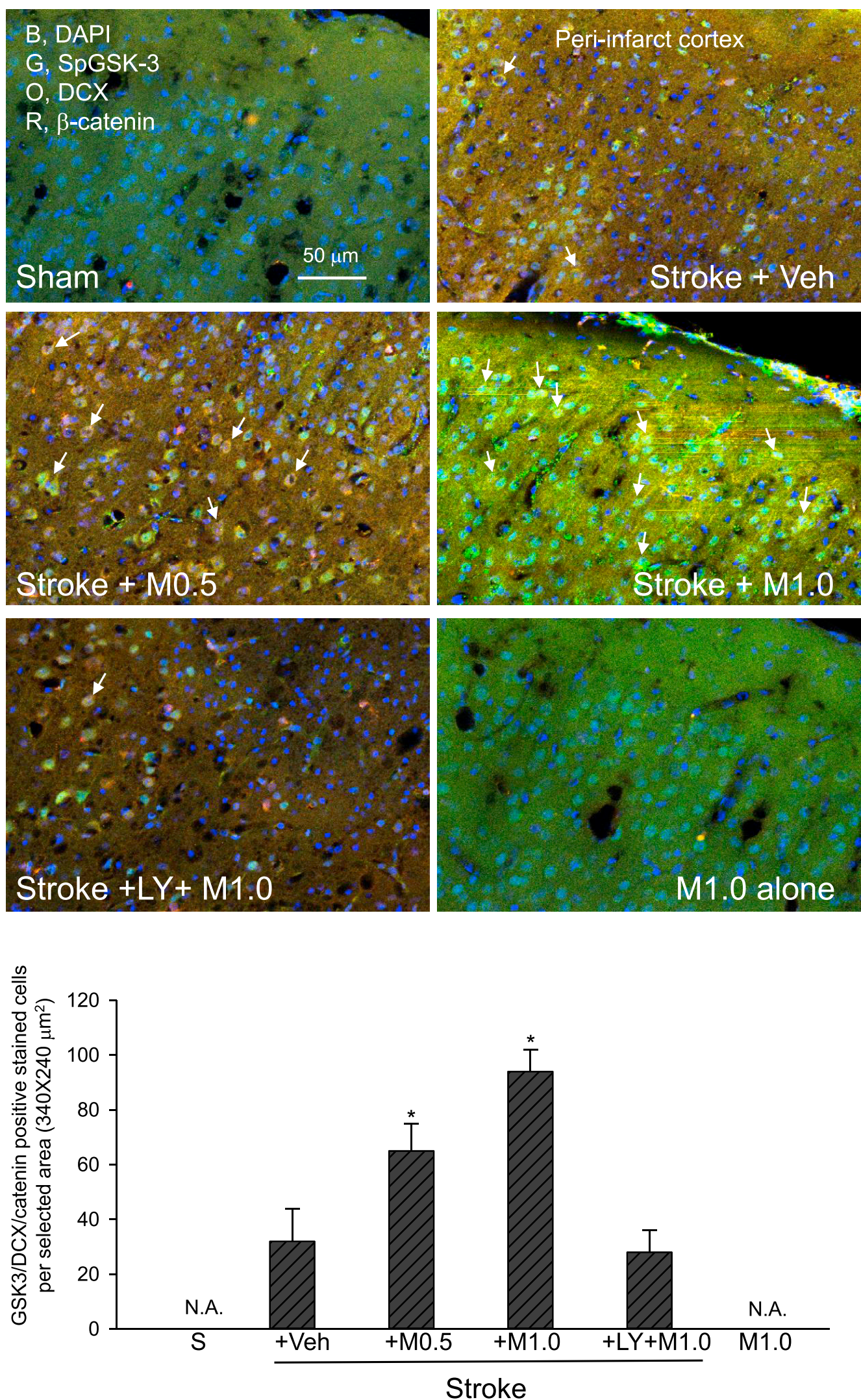

Fig. 5. (Continued). 
E
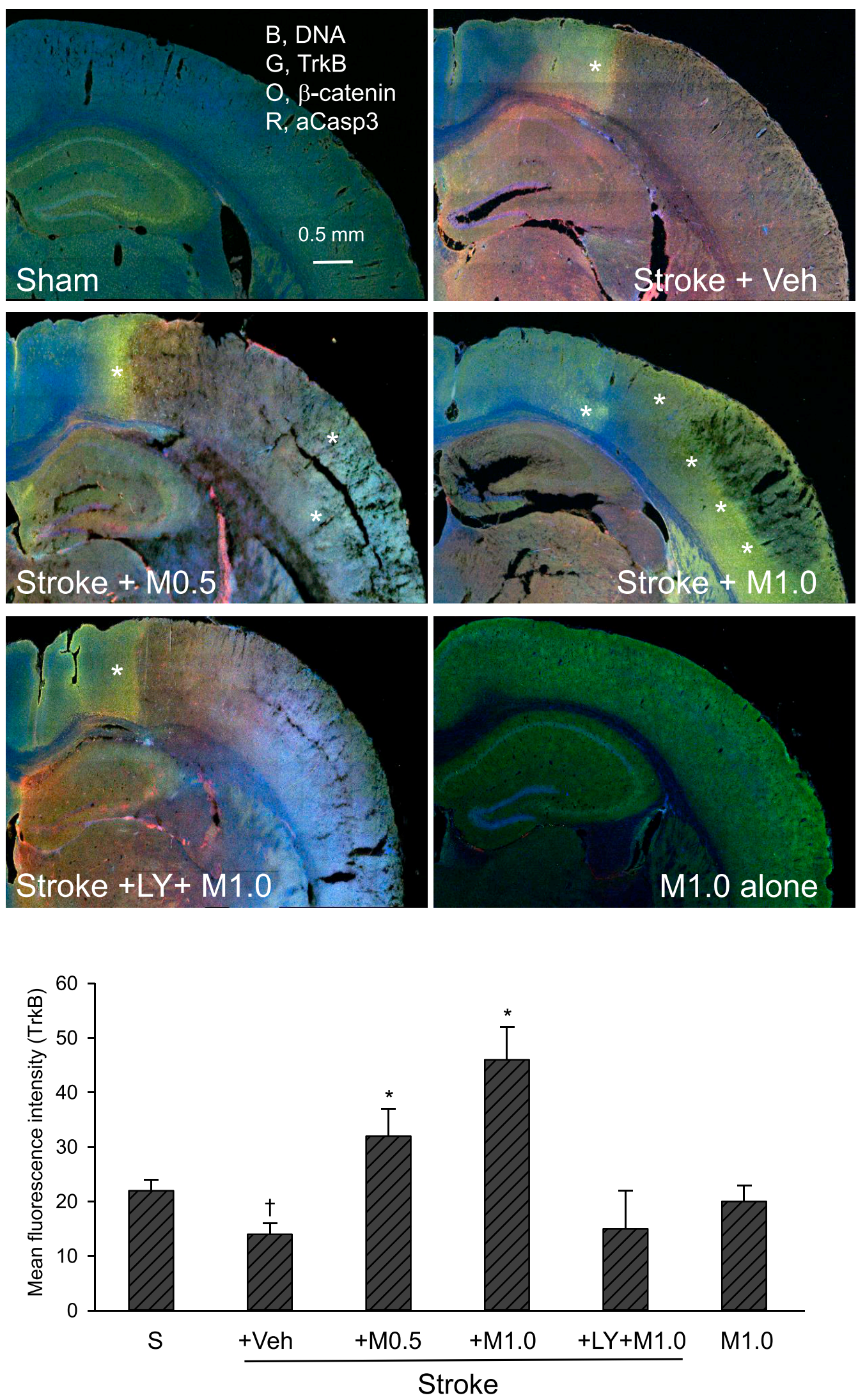

Fig. 5. (Continued). 
catenin pathway $[20,21]$. Our results indicated that ischemic stroke dramatically reduced the levels of Ser9-phosphorylated GSK-3 $\beta$ (SpGSK-3, an indicator for GSK-3 inactivation) within peri-infarct cortex (Fig. 5B) and dentate gurus (Fig. 5C), which correlated well with severe apoptosis in these areas (as indicated by arrows, Fig. 5B; Stroke + Veh vs. Sham, Fig. 5B, $p<0.05)$. In contrast, SpGSK-3 levels were significantly increased after medicarpin treatment, and the SpGSK-3 staining was colocalized with the staining of $\beta$-catenin and DCX in both dentate gyrus and protected cortex (as indicated by arrows, Fig. 5C and D, Stroke + Medicarpin (M0.5 or M1.0) vs. Stroke + Veh, $p<0.05$ ). Altogether, our data indicate the involvement of the GSK-3/ $\beta$-catenin pathway in medicarpin's neuroprotective mechanism.

\subsection{Medicarpin up-regulated TrkB in mice after ischemic stroke}

To determine the possible involvement of TrkB (a BDNF receptor) in medicarpin's neuroprotective effect, we inspected TrkB expression levels in whole brain slice. Our results indicated that TrkB expression in ischemic stroke group was promoted and reserved only around the peri-infarct area (Fig. 5E, Stroke + Veh, green $(G)$, as indicated by stars), but did not extend to most parts of the damaged area with severe apoptosis (Fig. 5E, Stroke + Veh, red (R), caspase 3$)$. In contrast, medicarpin $(0.5-1.0 \mathrm{mg} / \mathrm{kg}$, i.v.) strongly elevated the expression level of TrkB (green, Fig. 5E) all around the protected zone, which was also colocalized with the expression of $\beta$-catenin (orange (O), Fig. 5E), typically observed near the cortex (as indicated by stars) (Fig. 5E, Stroke + Medicarpin (M0.5 or M1.0) vs. Stroke + Veh, $\mathrm{p}<0.05)$. Thus, these results implicated that both TrkB and $\beta$-catenin-mediated signaling pathway were likely engaged by medicarpin to ameliorate ischemic damage.
3.6. The neuroprotective effect of medicarpin was PI3K/Akt-dependent in mice after ischemic stroke

The PI3K/Akt signaling pathway is eminent for its neuroprotective function [4-7]. To address the role of PI3K/Akt signaling in medicarpin's neuroprotective effect, we examined whether medicarpinmediated neuroprotection was sabotaged when the PI3K/Akt signaling pathway was annulled by LY294002 (a PI3K inhibitor) before medicarpin treatment $(1.0 \mathrm{mg} / \mathrm{kg})$. It is noteworthy that in mice with ischemic injury, LY294002 pretreatment nearly abolished all of the protective effects and neuroprotective signals elicited by medicarpin (Figs. 2-5, $p>0.05$, as compared to Stroke + Veh), supporting an essential role of the PI3K/Akt-mediated signaling in medicarpin's neuroprotective action. It was also noted that LY294002 treatment alone barely affected the survival rates, neurological behaviors, or histochemical staining in either normal mice (as compared to sham group) or stroke mice (as compared to Stroke + Veh group) (Supporting data).

We then performed Western blot analysis to monitor the expression levels of the PI3K/Akt signaling molecules in the brain following ischemia induction. We found that, as compared to the sham control, ischemic stroke potently downregulated occludin (an NADH oxidase and a component of BBB structure [25]) and S473-phosphorylated Akt (pAkt) (Fig. 6, top two panels), suggesting ischemic stroke caused BBB disruption and Akt inactivation, respectively. The stroke-mediated inactivation of Akt was further supported by a parallel downregulation of Akt-dependent serine 9-phosphorylation of GSK-3 $\beta$, which led to reduced $\beta$-catenin levels and a consequent decrease in the expression of Bcl-2 and BDNF, both of which are $\beta$-catenin's downstream targets $[22,26]$ (Fig. 6, Stroke + Veh vs. Sham, $p<0.05)$. Notably, besides rescuing occludin expression in stroke mice, medicarpin $(1.0 \mathrm{mg} / \mathrm{kg}$,

Fig. 5. Effects of medicarpin on the changes in the expression levels of protein markers 24 h after cerebral ischemia-reperfusional injury (stroke) by confocal image analysis. (A) BBB damage and inflammation. Upper panel, adopted from the ipsilateral ischemic hemisphere (bregma-1.5 to $-1.9 \mathrm{~mm}$ ) for assessing BBB and tissue damage (loss of occludin, green, $G$ ), inflammation (p65NF- $\kappa B$ expression (red, $R$, as arrows indicated), and DAPI (blue, B, nuclei); square boxes indicate peri-infarct cortex and dentate gyrus (DG) of hippocampus, respectively. Lower panel, statistical summary. (B) GSK-3 inactivation and apoptosis. Upper panel, adopted from peri-infarct cortex for detecting GSK-3 inactivation by the levels of phospho-S9-GSK-3 $\beta$ (SpGSK-3, green, G), apoptosis by the levels of activated caspase 3 (aCasp3, red, $R$, as arrows indicated). DAPI staining (blue, B) indicates the position of cell nuclei. Lower panel, statistical summary. (C) Neurogenesis marker. Upper panel, adopted from dentate gyrus (DG) of hippocampus for examining neurogenesis (doublecortin (DCX), orange, $\mathrm{O}$, as arrows indicated) and colocalization of GSK-3 inactivation (phosphoS9-GSK-3 $\beta$ (SpGSK-3), green, G), $\beta$-catenin expression (red, R), and DAPI (blue, B, nuclei). Lower panel, statistical summary. (D) GSK-3 inactivation, $\beta$-catenin expression and neurogenesis. Upper panel, adopted from peri-infarct cortex for colocalization (as arrows indicated) of GSK-3 inactivation (phospho-S9-GSK-3 $\beta$ (SpGSK-3), green, G), $\beta$-catenin expression (red, R), neurogenesis (doublecortin (DCX), orange, O), and DAPI (blue, B, nuclei). Lower panel, statistical summary. (E) TrkB expression. Upper, TrkB (green, G), $\beta$-catenin (orange, O), activated caspase 3 (aCasp3, red, R), and DAPI (blue, B, nuclei); stars indicated strong staining and colocalization of TrkB and $\beta$-catenin. Lower, statistical summary. Animal subgroups are described in Fig. 2. 

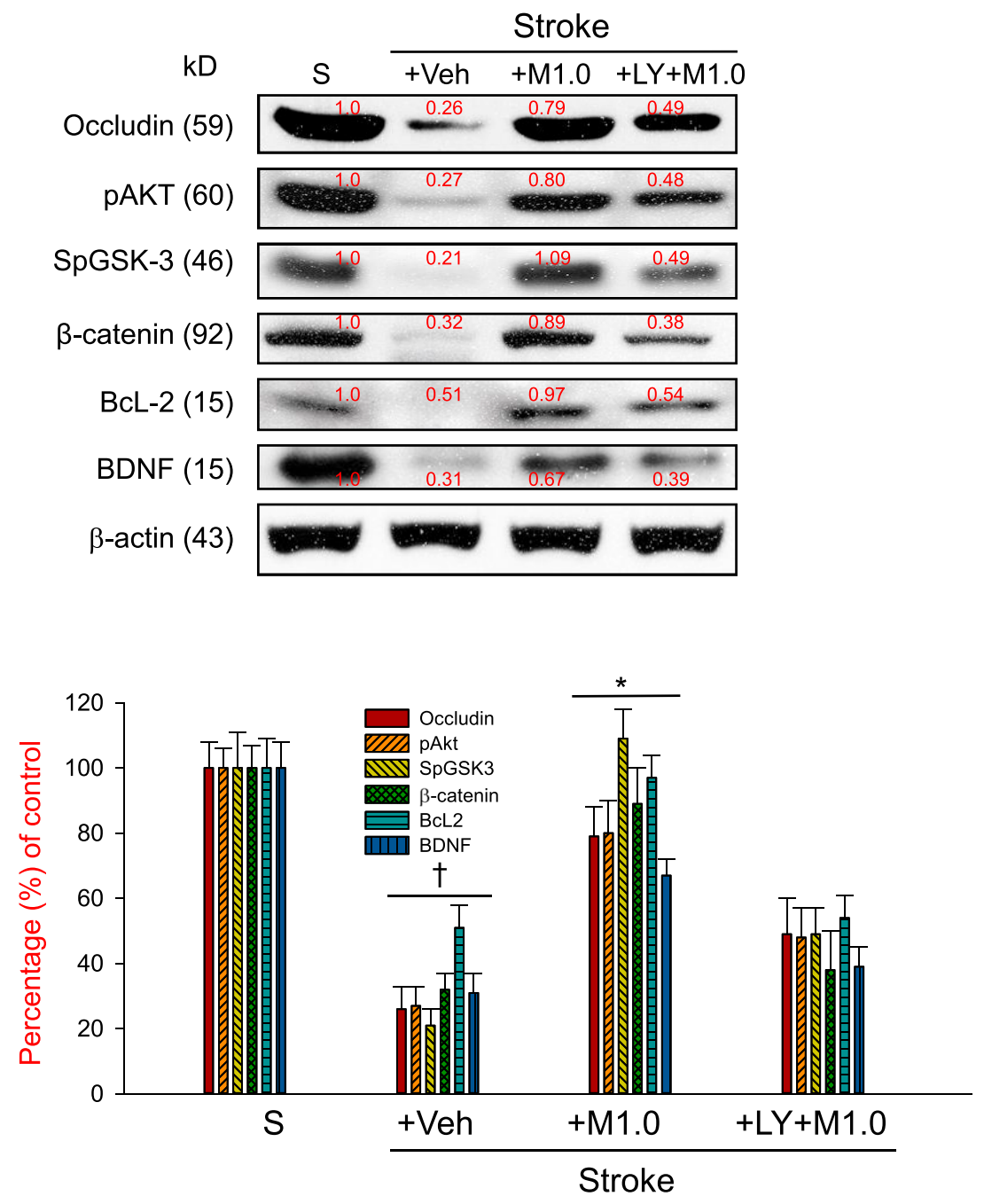

Fig. 6. Effects of medicarpin on the changes in the expression levels of proteins in neurogenesis-promoting-associated signaling $24 \mathrm{~h}$ after stroke injury. (A) Upper, typical Western blot analysis using tissues from the ischemic (ipsilateral) brain hemisphere was conducted to show the changes in the levels of occludin, phospho-Akt (pAkt), phospho-S9-GSK-3 $\beta$ (SpGSK-3), $\beta$-catenin, Bcl-2, and BDNF. $\beta$-actin was included as a reference for normalization. Lower, statistical results from the densitometric measurements after normalization against $\beta$-actin. Animal sub-groups are described in Fig. 2.

i.v.) enhanced the levels of Bcl-2 and BDNF, possibly through activating PI3K/Akt (as evidenced by increased pAkt levels) to mediate GSK-3 inactivation (as supported by increased SpGSK-3 levels) for upregulating $\beta$-catenin (Fig. 6, Stroke + Veh vs. Stroke + M1.0, $p<0.05)$. Nevertheless, medicarpininduced occludin expression and activation of the
PI3K/Akt/SpGSK-3/ $\beta$-catenin/Bcl-2; $\quad$ BDNF signaling axis were abrogated when pre-treating stroke mice with LY294002 (Fig. 6, $p>0.05$, as compared to Stroke + Veh). Overall, these lines of evidence supported that PI3K/Akt activity is triggered by medicarpin to protect mice from ischemic stroke-induced injury.

Table 1. Anti-apoptotic and anti-inflammatory effects of medicarpin in two respective cellular models.

\begin{tabular}{llll}
\hline & N2A/OGD apoptosis $\left(\mathrm{IC}_{50} \mu \mathrm{M}\right)$ & $\mathrm{BV} 2 / \mathrm{NO}\left(\mathrm{IC}_{50} \mu \mathrm{M}\right)$ & DPPH scavenging $\left(\mathrm{IC}_{50} \mu \mathrm{M}\right)$ \\
\hline Medicarpin & $13 \pm 2$ & $5 \pm 1$ & $>50$ \\
AR-A01 & $8 \pm 1$ & $\mathrm{ND}$ & ND \\
PDTC & $\mathrm{ND}$ & $12 \pm 1$ & ND \\
Trolox & $\mathrm{ND}$ & $\mathrm{ND}$ & $22 \pm 2$ \\
\hline
\end{tabular}

Oxygen-glucose deprivation (OGD)-induced Neuro 2 A (N2A) cell apoptosis and LPS-induced BV2 activation (NO production) were used to determine the anti-apoptosis and anti-inflammatory activities of medicarpin. Three positive control reagents were included: (1) AR-A01 (AR-A014418), a selective GSK-3 inhibitor, (2) PDTC (ammonium pyrrolidinedithiocarbamate), an NF- $\mathrm{BB}$ inhibitor and (3) trolox, a vitamin E analogue. ND, not determined; Data are presented as mean \pm SEM $(n=5)$. 

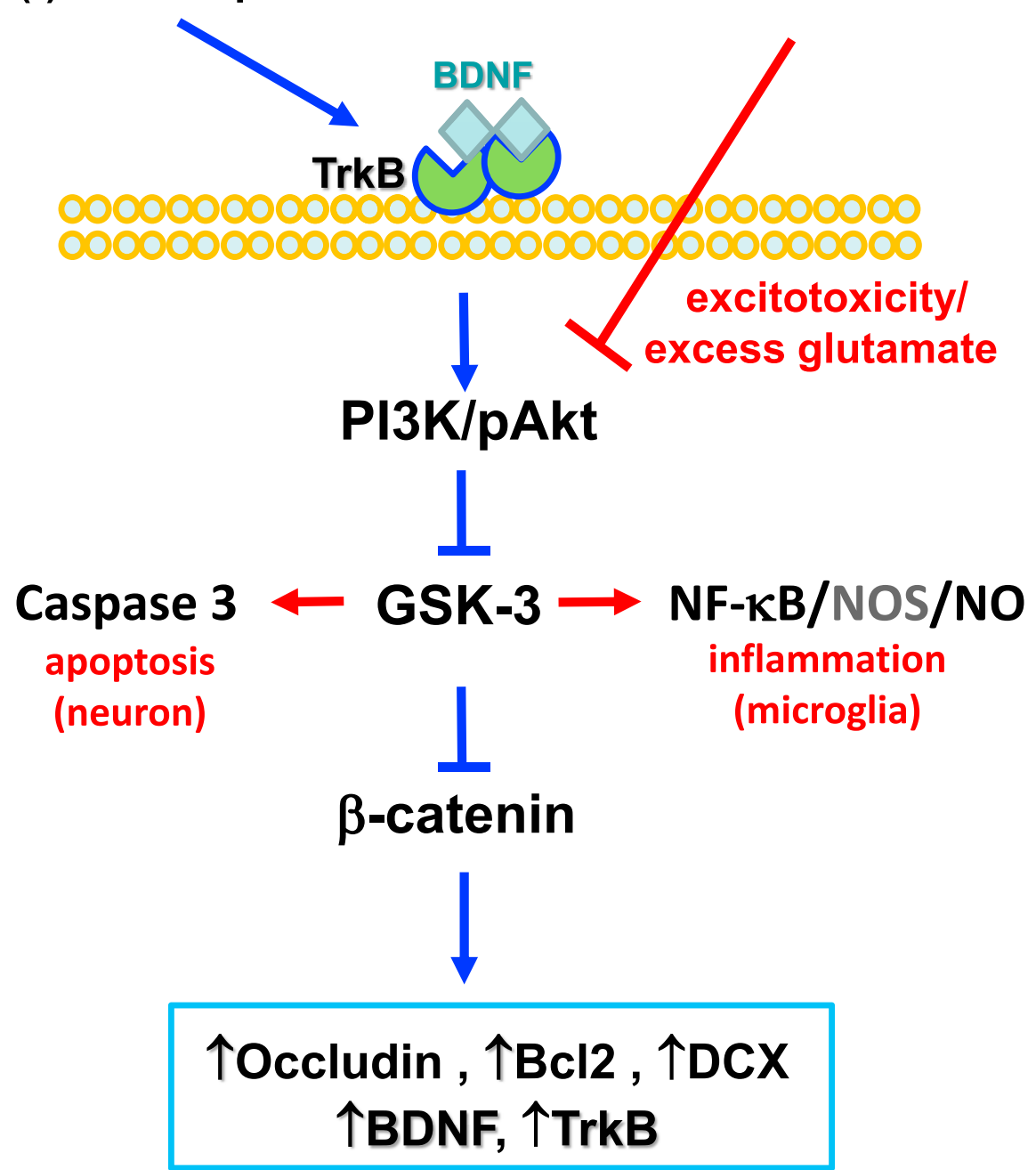

\section{Neuroprotection \& Neurogenesis promoting signaling by medicarpin}

Fig. 7. A schematic illustration showing the key pathways regulated by medicarpin for achieving its neuroprotective and neurogenesis-promoting effects. Ischemic stroke induced PI3K/Akt inhibition due to excess release of glutamate (excitotoxicity). Medicarpin triggers the activation of PI3K/Akt to inactivate GSK-3 for upregulating $\beta$-catenin, which then promotes neurogenesis by stimulating the expression of neuroprotective and neurogenesis proteins, including occludin, Bcl-2, DCX, BDNF, and TrkB.

\subsection{Neuroprotective and anti-inflammatory effects of medicarpin in vitro}

Our above in vivo examination demonstrated medicarpin suppressed ischemia stroke-induced neuronal damage and p65NF- $\kappa$ B activation (Figs. 4 and 5). To further substantiate the in vivo evidence, we explored medicarpin's neuroprotective and anti-inflammatory effects in the cellular models of OGD-induced neuronal cell apoptosis and LPS-mediated nitric oxide
(NO) production in microglial cell [4], respectively. As shown in Table 1, medicarpin exhibited anti-apoptotic activity against OGD-induced injury of Neuro 2A cells with an $\mathrm{IC}_{50}$ around $13 \pm 2 \mu \mathrm{M}$. As a positive control, AR-a014418 (a GSK-3 inhibitor) also inhibited OGDinduced apoptosis ( $\mathrm{IC}_{50}$ around $8 \pm 1 \mu \mathrm{M}$ ). In addition, we noticed medicarpin displayed a significant antiinflammatory effect, as evidenced by a marked reduction of LPS-induced nitric oxide (NO) production in BV-2 cells when co-treated with medicarpin. It 
is also noteworthy that medicarpin suppressed LPSinduced NO production with an $\mathrm{IC}_{50}$ of $5 \pm 1 \mu \mathrm{M}$, which was almost as potent as PDTC, an NF- $\kappa B$ inhibitor ( $\mathrm{IC}_{50}$ around $\left.12 \pm 1 \mu \mathrm{M}\right)$.

As anti-inflammatory effect is generally accompanied by anti-oxidative activity, we then addressed whether medicarpin alone displayed any anti-oxidative capacity using DPPH free radical scavenging assay [4]. Our results revealed that medicarpin alone barely showed free radical scavenging activity $\left(\mathrm{IC}_{50}>50 \mu \mathrm{M}\right.$ ) as compared to that of trolox (a vitamin $\mathrm{E}$ analogue), which displayed DPPH free radical scavenging activity with an $\mathrm{IC}_{50}$ of $22 \pm 2 \mu \mathrm{M}$ (Table 1 ).

Collectively, this is the first report to demonstrate that medicarpin, an active component isolated from the major herb (Radix Hedysari) in Bu-yang Huan-wu decoction (BHD), could effectively ameliorate ischemic stroke-associated brain damage by inhibiting the inflammatory factors/mediator (p65NF- $\kappa \mathrm{B}$, microglia/NO)- and apoptotic factor (activated caspase 3, neuronal cells/OGD)-related tissue/cell damage. Moreover, we proved that medicarpin promotes neuroprotection (occludin, Bcl2) and neurogenesis (BDNF/TrkB/DCX) by preserving PI3K/Akt activity under ischemic stroke-induced excitotoxicity. In turn, PI3K/Akt induces GSK-3 inactivation, leading to the activation of $\beta$-catenin-mediated signaling for initiating neuroprotection and neurogenesis. Our results further implicate that both the TCM remedy BHD and its primary herb Radix Hedysari could be promising agents for treating ischemic stroke, given their beneficial effects and the underlying mechanisms of action could be partially explained by their active component medicarpin.

GSK-3 is a neurological master switch protein [26]. Activation of GSK-3 activity has been reported to mediate inflammation and apoptosis during ischemic stroke $[11,20,21,27]$. In this study, we found that an inflammatory marker (NF- $\kappa \mathrm{B})$ and an apoptosis marker (activated caspase 3 ) were highly expressed in the ischemic stroke-damaged area, which was accompanied by GSK-3 activation (loss of SpGSK-3 staining) as previously reported by us [4,20-22]. Significantly, we herein demonstrated that medicarpin administration preserved the ischemic strokedamaged area by inducing GSK-3 inactivation along with $\beta$-catenin upregulation, which was accompanied by reduced BBB and tissue damage (loss of occludin) and decreased expression levels of the inflammatory marker (NF- $\kappa \mathrm{B}$ ) and apoptosis marker (activated caspase 3). In vitro study further supported that medicarpin displays potent anti-inflammation and antiapoptosis activities, as evidenced by medicarpin- mediated inhibition of microglial cell activation and OGD-induced neuronal cell apoptosis, respectively. These lines of evidence together argue that the antiinflammatory and anti-apoptotic effects are responsible for medicarpin's protective effect against ischemic stroke.

Many transcription factors implicated in neural development are regulated by GSK-3 [26,28]. Among these factors, $\beta$-catenin, a cofactor of the T-cell factor/ lymphoid enhancer factor (TCF/LEF) family of transcription factors, is degraded in the resting state by the ubiquitin-proteasome machinery due to GSK-3mediated phosphorylation. Inactivation of GSK-3 by Wnt/frizzled-associated proteins allows $\beta$-catenin accumulation $[29,30]$. It is well known that upregulation of $\beta$-catenin is critical to the control of progenitor proliferation in multiple regions of the developing nervous system [31,32], as well as in the dentate gyrus and lateral ventricle in brains with ischemic stroke $[20,21]$. Here, we observed that in the ischemic stroke group without medicarpin treatment, $\beta$-catenin levels were scarce in most of the ischemic stroke-damaged area, even though they were increased in the periinfarct boundary zone. In the medicarpin-treated group, in contrast, $\beta$-catenin was upregulated extensively almost all around the stroke-compromised area. This phenomenon was also consistent with GSK-3 inactivation and the upregulation of DCX (a marker for neural progenitors). Both signals (GSK-3 inactivation/DCX upregulation) were co-localized with $\beta$ catenin, especially near the dentate gyrus and protected cortex area.

Recently, many studies, including our previous reports, have revealed that BDNF plays a vital role in promoting neuronal plasticity, restructuring synapses associated with ischemia, and protecting brain tissues from ischemia-induced damages $[4,33]$. BDNF exerts an anti-apoptotic effect by stimulating the production of the anti-apoptotic Bcl-2 family proteins to exert neuroprotective effects [22,34]. BDNF is a novel target for improving cerebral ischemic/reperfusional injury and treating ischemic stroke [22,35]. Moreover, TrkB, a high-affinity receptor of BDNF, has been reported to reduce OGD-induced damage of the rat hippocampus [36] and diminish post-ischemic neuronal hyperexcitation and neurological deficits [37]. In the present study, we found that TrkB expression was colocalized with increased $\beta$-catenin expression in the peri-infarct boundary zone only but did not extend to most of the ischemic stroke-damaged area. In contrast, TrkB levels were increased extensively in the medicarpintreated group and spread to most of the strokecompromised regions. It is noteworthy that, in the 
brain with ischemia stroke, the aforementioned expression patterns of TrkB without or with medicarpin treatment were highly comparable to that of BDNF. Accordingly, it is plausible to argue that medicarpin exerts its neuroprotective action by engaging the BDNF/Trk-mediated neuroprotective/neurogenerative signaling pathway.

It is well-known that the PI3K/Akt signaling pathway is prominent in the regulation of cell survival and is stimulated after activation of TrkB receptors by BDNF [36]. Extensive research, including ours, has confirmed that the activation of the PI3K/Akt signaling pathway could inhibit the expression of apoptosisrelated proteins, leading to alleviation of cerebral ischemic/reperfusional injury $[4,38]$. Conversely, the protective effect of BDNF on cells can be blocked by the inhibitors of TrkB and PI3K [39]. In the present study, the level of pAkt was significantly decreased in the brains with ischemic stroke. Nevertheless, medicarpin treatment significantly upregulated pAkt, which was consistent with the enhanced levels of BDNF and TrkB. Notably, the aforementioned protective effects and signals elicited by medicarpin were all severely reduced by pretreatment with LY294002 (a PI3K inhibitor), supporting that PI3K/Akt was activated by medicarpin in this model.

\section{Conclusions}

We herein present the first report demonstrating the neuroprotective and reparative effects of medicarpin on mice after cerebral ischemic/reperfusional injury. Notably, medicarpin upregulates neuroprotective proteins such as Bcl-2 and DCX via activation of PI3K/Akt to induce GSK-3 inactivation. In turn, the inactivated GSK-3 fails to repress $\beta$-catenin, leading to the up-regulation of BDNF/TrkBdependent neurogenesis (Fig. 7).

\section{Authors' contributions}

C.M. Chern, Y.H. Wang, K.T. Liou and Y.C. Shen participated in research design., conducted the experiments and wrote or contributed to the writing of the manuscript. C.K. Lu, C.L. Chang and K.C. Tsai. Contributed reagents or analytic tools. C.C. Chang contributed to the revision of this work.

\section{Conflict of interest}

The authors declare that they have no conflict of interest.

\section{Acknowledgments}

This study was supported, in part, by grants from the Ministry of Science and Technology (MOST), R.O.C.; Division of Neurovascular Disease, Neurological Institute, Taipei Veterans General Hospital; School of Medicine, National Yang-Ming University, Taiwan; and the National Research Institute of Chinese Medicine, Taipei, Taiwan, awarded to Dr. Chern C.M., Dr. Liou K.T. and Dr. Shen Y.C. (MOST 108-2320-B-077003-MY3, MOHW109-NRICM-M-325-123401).

\section{Appendix Supplementary material}

S.1. Effects of LY294002 alone on survival rates and neurological deficits after cerebral ischemic/reperfusional injury in mice

Treatment of LY294002 (at $1.0 \mathrm{mg} / \mathrm{kg}$, i.p., $1.5 \mathrm{~h}$ after stroke, Stroke $+\mathrm{LY}, \mathrm{n}=5$ ) did not improve survival rate and neurological deficits after cerebral ischemic/reperfusional injury in mice (Fig. S1, $p>0.05)$, as compared to vehicle-treated group with ischemic stroke (Stroke + Veh, Figs. 2-3).

Treatment of LY294002 (at $1.0 \mathrm{mg} / \mathrm{kg}$, i.p., LY alone, $\mathrm{n}=5$ ) in normal mice also did not induce any significant change on survival rate and neurological deficits (Fig. S1, $p>0.05$ ), as compared to shamoperated group (Sham, Figs. 2-3).

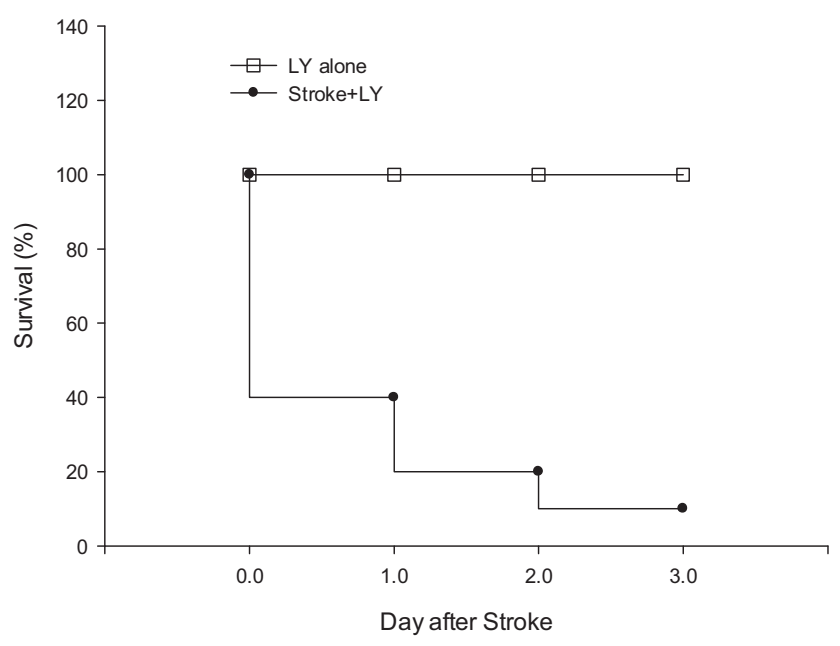

Fig. S1. Survival rates in LY alone and Stroke + LY:

Neurological deficits (moving distance (m); Area coverage $(\mathrm{A} \%))$ in LY alone and Stroke + LY: 


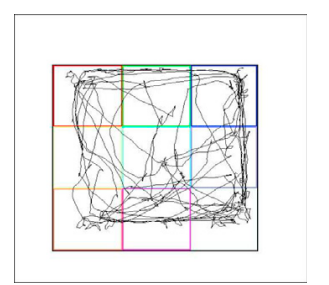

(LY alone: $26.0 \pm 1.8 \mathrm{~m} ;(\mathrm{A} \%) 89 \pm 1 \%$ )

S.2. Effects of LY294002 alone on cerebral infarction, $B B B /$ tissue damage, inflammation, and apoptosis after cerebral ischemic/reperfusional injury in mice

Treatment of LY294002 (at $1.0 \mathrm{mg} / \mathrm{kg}$, i.p., $1.5 \mathrm{~h}$ after stroke; Stroke $+\mathrm{LY}, \mathrm{n}=5$ ) did not improve cerebral infarction (Fig. S2, TTC stain, $p>0.05$ ), $\mathrm{BBB} /$ tissue damage (occludin, green), inflammation (p65NFкB upexpression and nuclei translocation, purple), and apoptosis (cleaved/activated caspase 3 expression (aCap3), red) after cerebral ischemic/ reperfusional injury in mice (Fig. S2), as compared to vehicle-treated group with ischemic stroke (Stroke + Veh, Figs. 4, 5A and B).

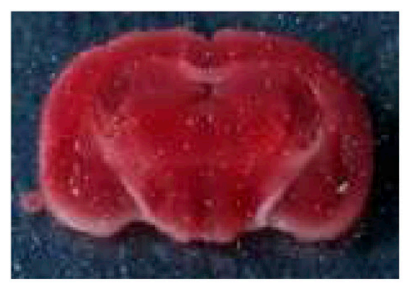

(LY alone: $0 \pm 0 \mathrm{~mm}^{3}$ )

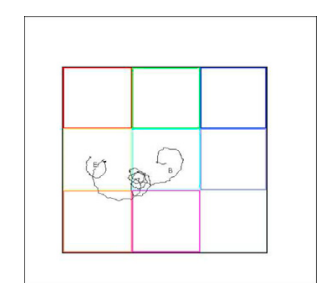

(Stroke+LY: $4.3 \pm 2.3 \mathrm{~m} ;(\mathrm{A} \%) 30 \pm 3 \%$ )

Treatment of LY294002 (at $1.0 \mathrm{mg} / \mathrm{kg}$, i.p., LY alone) in normal mice also did not induce significant change on cerebral infarction (Fig. S2, $p>0.05$ ), inflammation, and apoptosis (Fig. S2), as compared to sham-operated group (Figs. 4, 5A and B).

$\mathrm{BBB} /$ tissue damage (occludin, green, arrow) and inflammation ( $\mathrm{p} 65 \mathrm{NF} \kappa \mathrm{B}$ upexpression and nuclei translocation, purple, arrow head) in LY alone and Stroke + LY.

$\mathrm{BBB} /$ tissue protection (SpGSK-3, green, arrow) and apoptosis (cleaved/activated caspase 3 (aCasp3) expression, red, arrow head) in LY alone and Stroke + LY.

Fig. S2. Cerebral infarction (TTC stain, infarct volume $\left.\left(\mathrm{mm}^{3}\right)\right)$ in LY alone and Stroke + LY.
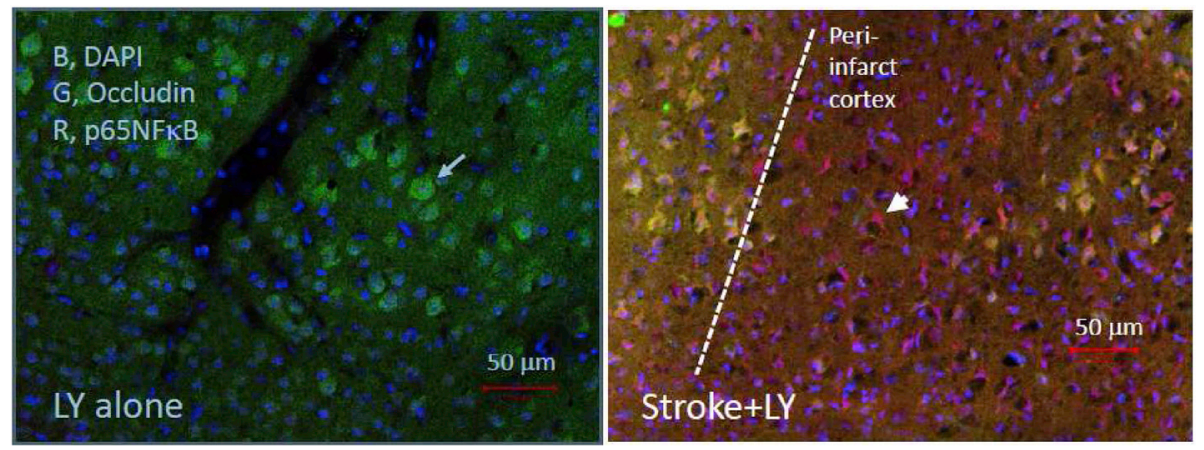


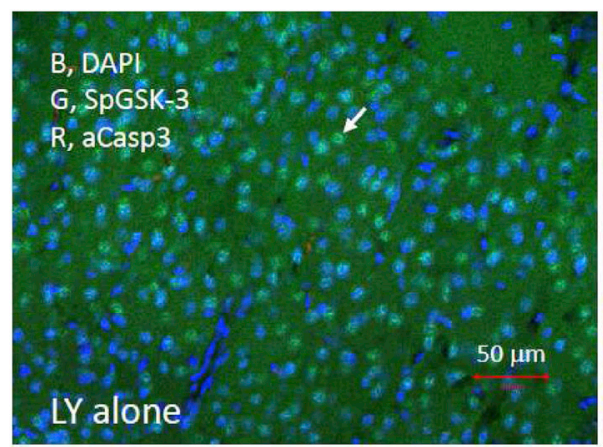

S.3. Effects of LY294002 alone on endogenous neurogenesis (DCX expression), GSK-3 inhibition (SpGSK3) and $\beta$-catenin upexpression after cerebral ischemicl reperfusional injury in mice

Treatment of LY294002 (at $1.0 \mathrm{mg} / \mathrm{kg}$, i.p., $1.5 \mathrm{~h}$ after stroke; Stroke + LY) did not modulate endogenous neurogenesis (DCX expression), GSK3 inhibition (SpGSK3) and $\beta$-catenin expression after cerebral ischemic/reperfusional injury in mice (Fig. S3) as compared to vehicle-treated group with ischemic stroke (Stroke + Veh, Fig. 5D).

Treatment of LY294002 (at $1.0 \mathrm{mg} / \mathrm{kg}$, i.p., LY alone) in normal mice also did not induce significant change on endogenous neurogenesis (DCX expression), GSK-3 inhibition and $\beta$-catenin expression (Fig. S3), as compared to sham-operated group (Sham, Fig. 5D).

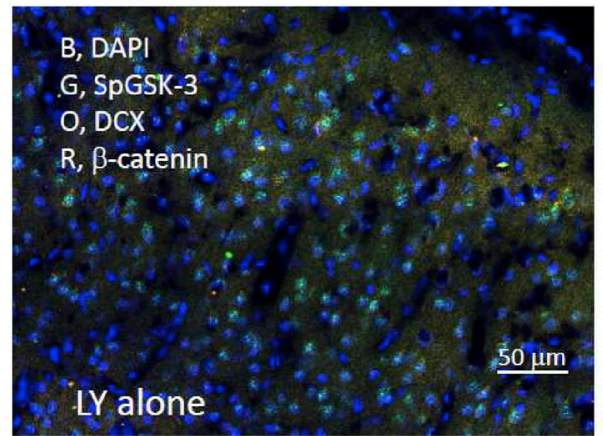

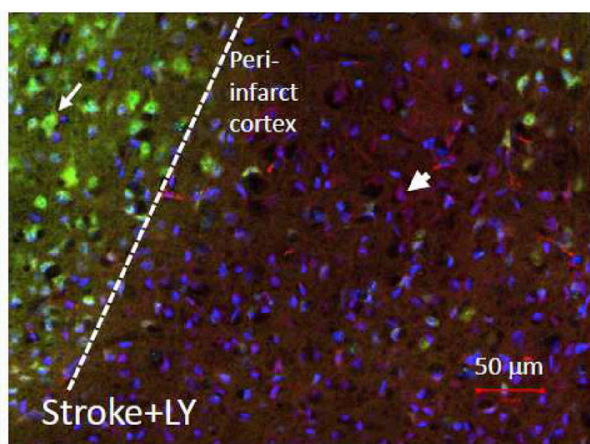

S.4. Effects of LY294002 alone on the expression of neurotrophic factor/receptor TrkB and upstream signaling molecule $\beta$-catenin after cerebral ischemicl reperfusional injury in mice

Treatment of LY294002 (at $1.0 \mathrm{mg} / \mathrm{kg}$, i.p., $1.5 \mathrm{~h}$ after stroke, Stroke + LY) did not modulate the expression of neurotrophic factor/receptor TrkB (green, G) and upstream signaling molecule $\beta$-catenin (orange, $\mathrm{O}$ ) or apoptosis (activated caspase 3 (aCasp3, red)) after cerebral ischemic/reperfusional injury in mice (Fig. S4), as compared to vehicle-treated group with ischemic stroke (Stroke + Veh, Fig. 5E).

Treatment of LY294002 (at $1.0 \mathrm{mg} / \mathrm{kg}$, i.p., LY alone) in normal mice also did not induce significant change on the expression of neurotrophic factor/ receptor TrkB (green) and upstream signaling molecule $\beta$-catenin (orange, $\mathrm{O}$ ) or apoptosis (activated caspase 3 (aCasp3, red)) (Fig. S4, LY alone), as compared to sham-operated group (Sham, Fig. 5E).

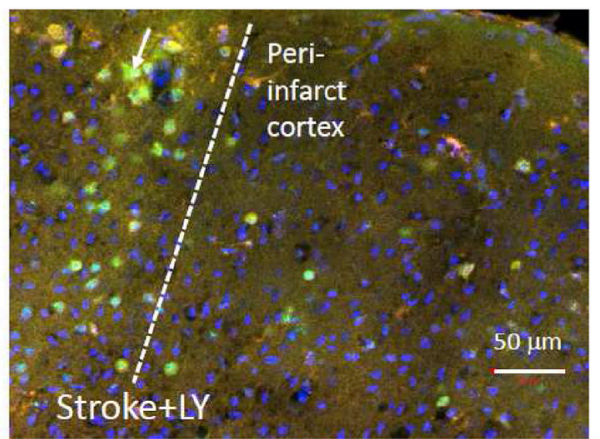

Fig. S3. Endogenous neurogenesis (DCX expression, orange, O), GSK-3 inhibition (SpGSK3, green, G) and $\beta$-catenin (red, R) upexpression in LY alone and Stroke $+L Y$; arrow indicated colocalization of SpGSK3, $\beta$-catenin and DCX at ischemic stroke preserved cortex area. 

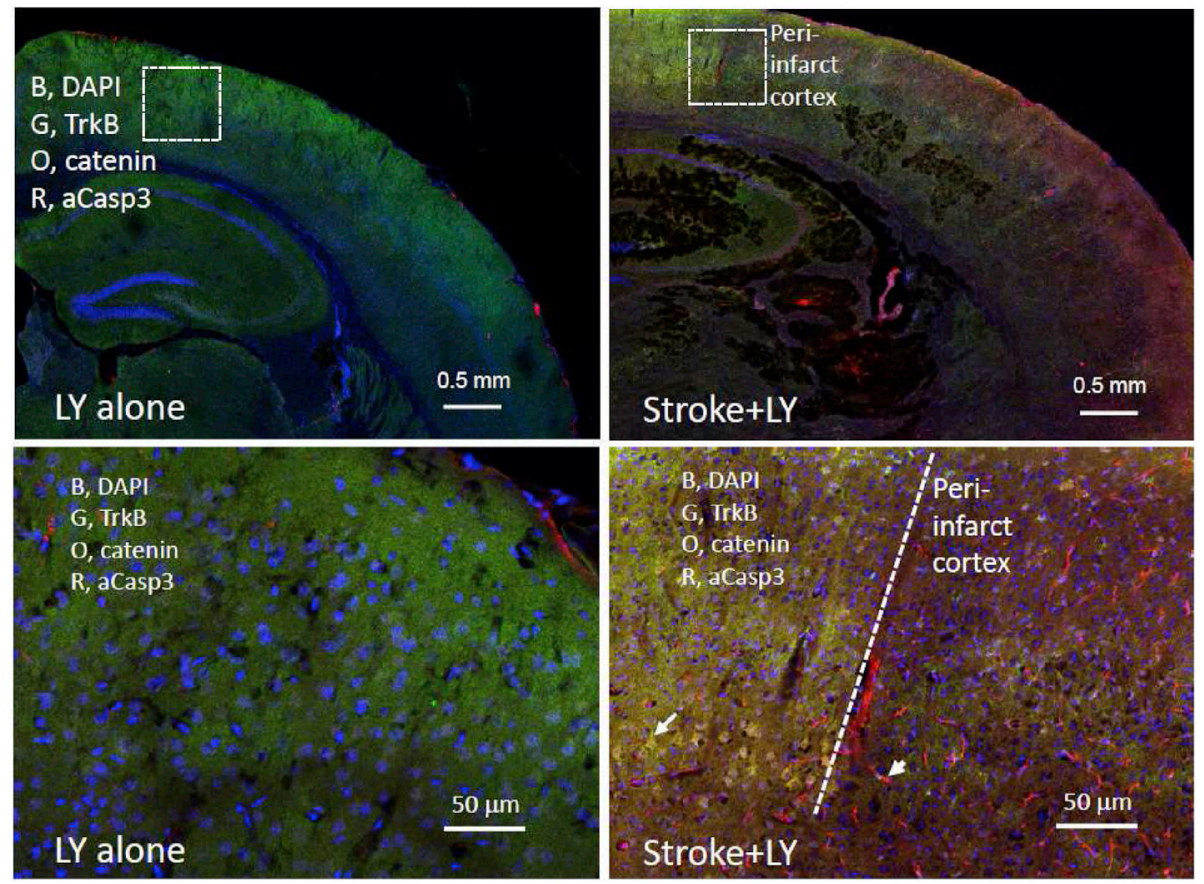

Fig. S4. Expression of neurotrophic factor/receptor TrkB (green, arrow) and colocalization with upstream signaling molecule $\beta$-catenin (orange, arrow), and apoptosis maker (aCasp3, red, arrow head); lower panel was adopted from the upper panel at peri-infarct cortex (square box). 

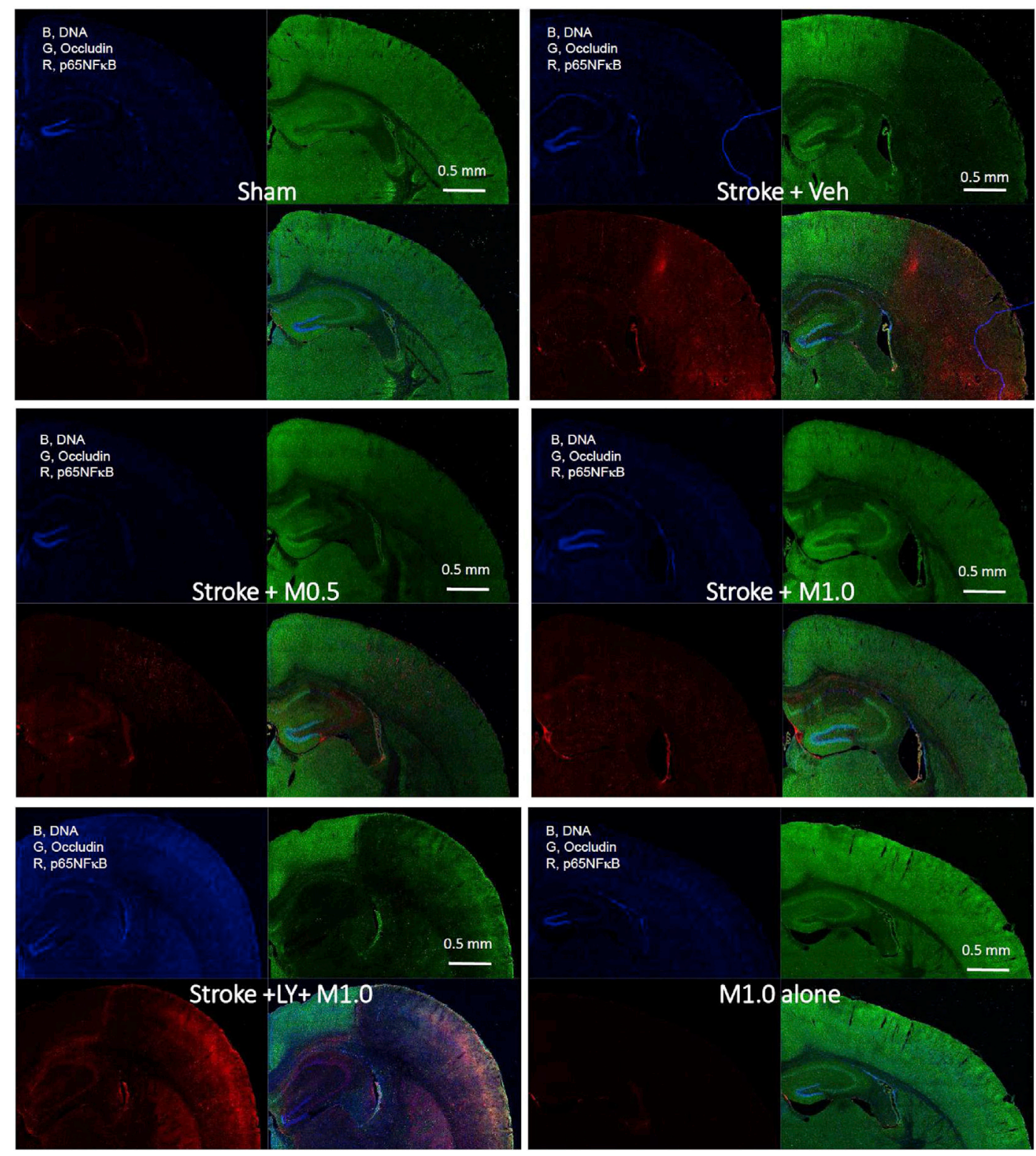

Fig. S5. Three independent color images adopted from Fig. 5A. 


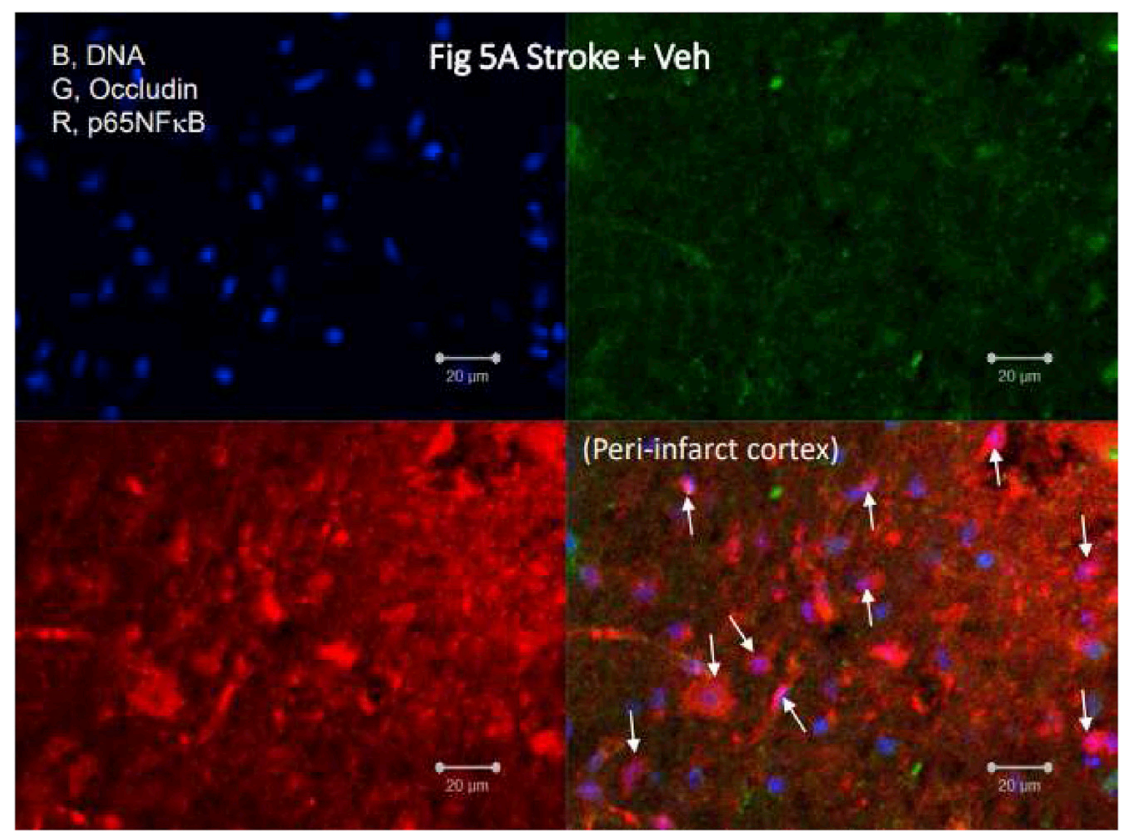

Fig. S6. Image adopted from Fig. 5A (Stroke + Veh) group, showing that p65NFאB (red) was colocalized with DAPI (nuclei, blue) near the peri-infarct cortex and turned into purple color showing below (as arrows indicated):

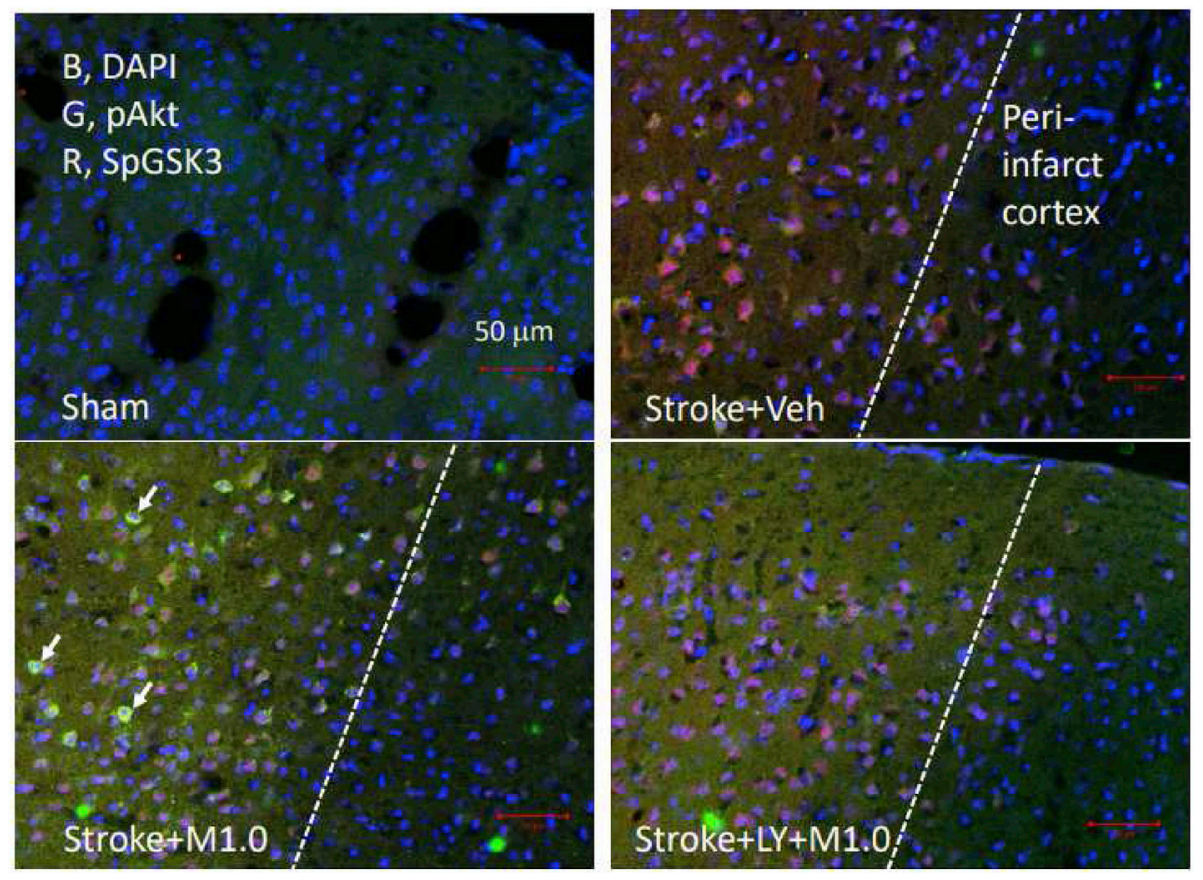

Fig. S7. Imaging showing that in the medicarpin's (1.0 mg/kg, M1.0) preserved cortex of the ischemic stroke group (Stroke + M1.0), the pAkt (green) was colocalized (as arrows indicated) with its substrate protein SpGSK3 (red), which was absent in other groups (Sham and Stroke + Veh), and was compromised by pretreatment with LY294002 (Stroke + LY + M1.0): 


\section{References}

[1] Katan M, Luft A. Global burden of stroke. Semin Neurol 2018;38:208-11.

[2] Zhou Z, Lu J, Liu WW, Manaenko A, Hou X, Mei Q, et al. Advances in stroke pharmacology. Pharmacol Ther 2018;191: 23-42.

[3] Pena ID, Borlongan C, Shen G, Davis W. Strategies to extend thrombolytic time window for ischemic stroke treatment: an unmet clinical need. J Stroke 2017;19:50-60.

[4] Wang YH, Chern CM, Liou KT, Kuo YH, Shen YC. Ergostatrien-7,9(11),22-trien-3beta-ol from Antrodia camphorata ameliorates ischemic stroke brain injury via downregulation of p65NF-kappa-B and caspase 3, and activation of Akt/ GSK3/catenin-associated neurogenesis. Food Funct 2019;10: 4725-38.

[5] Hou Y, Wang K, Wan W, Cheng Y, Pu X, Ye X. Resveratrol provides neuroprotection by regulating the JAK2/STAT3/ $\mathrm{PI} 3 \mathrm{~K} / \mathrm{AKT} / \mathrm{mTOR}$ pathway after stroke in rats. Genes Dis 2018;5:245-55.

[6] Wang MM, Zhang M, Feng YS, Xing Y, Tan ZX, Li WB, et al. Electroacupuncture inhibits neuronal autophagy and apoptosis via the PI3K/AKT pathway following ischemic stroke. Front Cell Neurosci 2020;14:134.

[7] Zhang W, Wu Y, Chen H, Yu D, Zhao J, Chen J. Neuroprotective effects of SOX5 against ischemic stroke by regulating VEGF/PI3K/AKT pathway. Gene 2021;767: 145-8.

[8] Chalecka-Franaszek E, Chuang DM. Lithium activates the serine/threonine kinase Akt-1and suppresses glutamateinduced inhibition of Akt-1 activity in neurons. Proc Natl Acad Sci USA 1999;96:8745-50.

[9] Rana AK, Singh D. Targeting glycogen synthase kinase-3 for oxidative stress and neuroinflammation: opportunities, challenges and future directions for cerebral stroke management. Neuropharmacology 2018;139:124-36.

[10] Dajani R, Fraser E, Roe SM, Young N, Good V, Dale TC, et al. Crystal structure of glycogen synthase kinase $3 \beta$ : structural basis for phosphate-primed substrate specificity and autoinhibition. Cell 2001;105:721-32.

[11] Chuang DM, Wang Z, Chiu CT. GSK-3 as a target for lithium-induced neuroprotection against excitotoxicity in neuronal cultures and animal models of ischemic stroke. Front Mol Neurosci 2011;4:15.

[12] Kasemeier-Kulesa JC, Morrison JA, Lefcort F, Kulesa PM. TrkB/BDNF signalling patterns the sympathetic nervous system. Nat Commun 2015;6:8281.

[13] Ni GX, Liang C, Wang J, Duan CQ, Wang P, Wang YL. Astragaloside IV improves neurobehavior and promotes hippocampal neurogenesis in MCAO rats though BDNFTrkB signaling pathway. Biomed Pharmacother 2020;130: 110353.

[14] Hargreaves JA, Mansfield JW, Coxon DT. Identification of medicarpin as a phytoalexin in the broad bean plant (Vicia Jaba L.). Nature 1976;262:318-9.

[15] Wang HY, Li T, Ji R, Xu F, Liu GX, Li YL, et al. Metabolites of medicarpin and their distributions in rats. Molecules 2019;24: 1966.

[16] Liu Y, Chen HB, Zhao YY, Wang B, Zhang QY, Zhang L, et al. Quantification and stability studies on the flavonoids of Radix Hedysari. J Agric Food Chem 2006;54:6634-9.

[17] Wang HW, Liou KT, Wang YH, Lu CK, Lin YL, Lee IJ, et al. Deciphering the neuroprotective mechanisms of Bu-yang Huan-Wu decoction by an integrative neurofunctional and genomic approach in ischemic stroke mice. J Ethnopharmacol 2011;138:22-33.

[18] Chang LC, Gerhäuser C, Song L, Farnsworth NR, Pezzuto JM, Kinghorn AD. Activity-guided isolation of constituents of Tephrosia purpurea with the potential to induce the phase II enzyme, quinone reductase. J Nat Prod 1997;60: 869-73.
[19] Care Gft, Animals UoL. National research council (US) committee for the update of the Guide for the Care and use of laboratory animals. Washington (DC): National Academies Press; 2011.

[20] Chern CM, Liao JF, Wang YH, Shen YC. Melatonin ameliorates neural function by promoting endogenous neurogenesis through the MT2 melatonin receptor in ischemicstroke mice. Free Radic Biol Med 2012;52:1634-47.

[21] Chern CM, Wang YH, Liou KT, Hou YC, Chen CC, Shen YC. 2Methoxystypandrone ameliorates brain function through preserving BBB integrity and promoting neurogenesis in mice with acute ischemic stroke. Biochem Pharmacol 2014;87:502-14.

[22] Chien MY, Chuang CH, Chern CM, Liou KT, Liu DZ, Hou YC, et al. Salvianolic acid A alleviates ischemic brain injury through the inhibition of inflammation and apoptosis and the promotion of neurogenesis in mice. Free Radic Biol Med 2016;99:508-19.

[23] Stroke Therapy Academic Industry R. (STAIR). Recommendations for standards regarding preclinical neuroprotective and restorative drug development. Stroke 1999;12:2752-8.

[24] Kim HH, Sawada N, Soydan G, Lee HS, Zhou Z, Hwang SK, et al. Additive effects of statin and dipyridamole on cerebral blood flow and stroke protection. J Cerebr Blood Flow Metabol 2008;28:1285-93.

[25] Castro V, Skowronska M, Lombardi J, He J, Seth N, Velichkovska $M$, et al. Occludin regulates glucose uptake and ATP production in pericytes by influencing AMP-activated protein kinase activity. J Cerebr Blood Flow Metabol 2018;38:317-32.

[26] Kim WY, Wang X, Wu Y, Doble BW, Patel S, Woodgett JR, et al. GSK-3 is a master regulator of neural progenitor homeostasis. Nat Neurosci 2009;12:1390-7.

[27] Wang YH, Liou KT, Tsai KC, Liu HK, Yang LM, Chern CM, et al. GSK-3 inhibition through GLP-1R allosteric activation mediates the neurogenesis promoting effect of P7C3 after cerebral ischemic/reperfusional injury in mice. Toxicol Appl Pharmacol 2018;357:88-105.

[28] Hur EM, Zhou FQ. GSK3 signalling in neural development. Nat Rev Neurosci 2010;11:539-51.

[29] Metcalfe C, Bienz M. Inhibition of GSK3 by Wnt signallingtwo contrasting models. J Cell Sci 2011;124:3537-44.

[30] Wu D, Pan W. GSK3: a multifaceted kinase in Wnt signaling. Trends Biochem Sci 2010;35:161-8.

[31] Woodhead GJ, Mutch CA, Olson EC, Chenn A. Cell-autonomous beta-catenin signaling regulates cortical precursor proliferation. J Neurosci 2006;26:12620-30.

[32] Gulacsi AA, Anderson SA. Beta-catenin-mediated Wnt signaling regulates neurogenesis in the ventral telencephalon. Nat Neurosci 2008;11:1383-91.

[33] Chai L, Guo H, Li H, Wang S, Wang YL, Shi F, et al. Scutellarin and caffeic acid ester fraction, active components of Dengzhanxixin injection, upregulate neurotrophins synthesis and release in hypoxia/reoxygenation rat astrocytes. J Ethnopharmacol 2013;150:100-7.

[34] Zhu G, Liu Y, Zhi Y, Jin Y, Li J, Shi W, et al. PKA- and $\mathrm{Ca}(2+)$-dependent p38 MAPK/CREB activation protects against manganese-mediated neuronal apoptosis. Toxicol Lett 2019;309:10-9.

[35] Luo L, Li C, Du X, Shi Q, Huang Q, Xu X, et al. Effect of aerobic exercise on BDNF/proBDNF expression in the ischemic hippocampus and depression recovery of rats after stroke. Behav Brain Res 2019;362:323-31.

[36] Tecuatl C, Herrrera-Lopez G, Martin-Avila A, Yin B, Weber S, Barrionuevo G, et al. TrkB-mediated activation of the phosphatidylinositol-3-kinase/Akt cascade reduces the damage inflicted by oxygen-glucose deprivation in area CA3 of the rat hippocampus. Eur J Neurosci 2018;47:1096-109.

[37] Chang LH, Lin HC, Huang SS, Chen IC, Chu KW, Chih CL, et al. Blockade of soluble epoxide hydrolase attenuates postischemic neuronal hyperexcitation and confers resilience against stroke with TrkB activation. Sci Rep 2018;8:118. 
[38] Tian X, An R, Luo Y, Li M, Xu L, Dong Z. Tamibarotene improves Hippocampus injury induced by focal cerebral ischemia-reperfusion via modulating PI3K/Akt pathway in rats. J Stroke Cerebrovasc Dis 2019;28:1832-40.
[39] Bai L, Zhang S, Zhou X, Li Y, Bai J. Brain-derived neurotrophic factor induces thioredoxin-1 expression through TrkB/Akt/CREB pathway in SH-SY5Y cells. Biochimie 2019; 160:55-60. 